\title{
Text S1:
}

\section{Congruence value $\left(V_{c}\right)$ : A measure to evaluate the similarity between two (phylogenetic) trees}

\author{
Shamim Ahmed and Koichi Nishigaki
}

\section{Calculation of the congruence value between tree-A and tree-B is described hereafter.}

\section{Definitions of terms used here}

The following terms were defined to determine the congruence value between two trees.

- Element: Each species is considered as a single element (SE) in this study. When couples of single elements formed a cluster (group), they are termed as cluster-elements (CE)

- Cluster: is a term given to a set of elements stemmed from a common root. A cluster can be composed of sub-clusters and single elements.

- Level: A tree consists of different hierarchies of branches beginning from the bottom to the top level. The top level is identical to so-called "root". In the definition adopted here, "level" is used to express the hierarchy of each element or cluster. A cluster positioned at level $\ell$ always contains one or more cluster(s) positioned at level $\ell-1$ by definition.

- Sub-cluster: A subset of a cluster consisting of more than or equal to two elements. A cluster of $n$ elements has various kinds of sub-clusters, say, if $\mathrm{n}=5 ; 5$ kinds of 4-membered sub-clusters $\left(={ }^{5} \mathrm{C}_{4}\right), 10$ kinds of 3 -membered sub-clusters $\left(={ }^{5} \mathrm{C}_{3}\right)$, and 10 kinds of 2-membered sub-clusters $\left(={ }^{5} \mathrm{C}_{2}\right)$.

- Cluster matching score (CMS): A score to evaluate the congruence between two clusters, which take into account the number of corresponding elements and the level difference of cluster.

- Phylogenetic tree/Tree: is a branching diagram showing the relationships of similarities or differences among various biological species (or elements) based on phenotypic and/or genotypic characteristics.

- Subject tree and Object tree: When the congruence value between two trees is to be calculated, the trees are allotted as a subject tree and object one for the sake of convenience. If the tree- $\mathrm{A}$ is considered as the subject, then the tree-B will be treated as the object and vice versa.

\section{Procedures:}

Assigning matching clusters: In this study we assigned matching clusters (or sub-clusters) for relevant trees with a common cluster name based on the following criteria.

Criterion 1. If all elements in a cluster belonging to the subject tree-A correspond to the elements of a particular cluster in the object tree, a same name is assigned to both of the clusters like $C_{X}$ (see Panel 1b). 
Criterion 2. If more than 50\% elements of a cluster of the subject tree-A correspond to some of the elements of a single cluster belonging to the object tree $\mathrm{B}$, a same yet discriminative name is assigned as $\mathrm{C}_{\mathrm{X}}$ for the cluster of the subject tree and $\mathrm{C}_{\mathrm{X}}$ ' for the cluster belonging to the tree $\mathrm{B}$.

Criterion 3. No name is given if $\leq 50 \%$ elements of a cluster of the subjective tree can find the corresponding elements in any cluster contained in the objective tree.

\section{How to calculate cluster matching score (CMS):}

\section{For $\mathbf{C}_{\mathrm{x}}$ cluster (criterion 1):}

$\mathrm{CMS}$ is the number of elements at level $\ell-1$ contained in $\mathrm{C}_{\mathrm{x}}$ cluster which is at level $\ell$. i.e.,

$$
\mathrm{CMS}_{\mathrm{Cx}}=\text { Number of elements in } \mathrm{C}_{\mathrm{x}} \text { cluster }
$$

If the cluster of interest contains the lower level elements than level $\ell-1$, then those elements are scored with the reduction rate $\gamma$;

$$
\gamma=(1 / 2)^{(\ell-1)-\ell^{\prime}}=(1 / 2)^{\left(\ell-\ell^{\prime}\right)-1}=(1 / 2)^{\Delta \ell-1}
$$

where $\ell, \ell^{\prime}$ and $\Delta \ell$ represent the level for the cluster of interest (of which CMS is calculated), level of the lower hierarchy cluster/element and the level difference, respectively.

Therefore, in such case, CMS will be expressed as follows;

$$
\mathrm{CMS}_{\mathrm{Cx}}=\Sigma \text { each element in Cx cluster } \mathrm{x}(1 / 2)^{\Delta \ell-1}
$$

\section{For $\mathbf{C}_{\mathrm{x}}$ ' cluster (criteria 2):}

When a sub-cluster is involved, the following consideration is made to calculate the CMS:

As an $m$-membered sub-cluster within a cluster of $n$ elements $(n \geq m)$ can appear in the number of states $S$ (only single and same level elements are considered);

$$
S={ }^{n} \mathrm{C}_{m}=\frac{n !}{m !(m-n) !}
$$

One possible way to consider this number of degeneracy in the calculation of CMS of $\mathrm{C}_{\mathrm{x}}$ ' cluster is to divide by this number $S$ :

$$
\mathrm{CMS}_{\mathrm{Cx}},=\frac{1}{s} \mathrm{x} \text { Number of elements of } \mathrm{Cx}^{\prime} \text { cluster }
$$

When a higher level cluster is assigned as $\mathrm{C}_{\mathrm{x}}$, which is composed of single and/or cluster elements positioned at level $\ell-1$ and/or lower than level $\ell-1$, in such cases CMS of $C_{\mathrm{x}}$ ' cluster will be expressed as follows;

$$
\mathrm{CMS}_{\mathrm{Cx}}{ }^{\prime}=\Sigma \text { each element in } \mathrm{C}_{\mathrm{x}}{ }^{\prime} \text { cluster } \mathrm{x}(1 / 2)^{\Delta \ell-1}
$$


Definition of Branch length and number: If a cluster positioned at level $\ell$ and contains elements with a length more than $1(\ell-(\ell-1)=1$ unit, the branch length corresponding to the difference in the level), then the length of that particular branch (>1 length, marked as red in Panel 1c) will be considered as;

$$
\text { Branch length }=(1 / 2)^{\Delta \ell-1} \text { (unit), }
$$

where $\Delta \ell$ represent the level difference of a branch. If the $\Delta \ell>4$ of a branch, it has been ignored in this study for the convenience.

\section{Definition of congruence value $\left(V_{c}\right)$ :}

The definition of the congruence value is:

$V_{\mathrm{c}}=\left(\sum \mathrm{CMS}\right.$ over trees $\mathrm{A}$ and $\mathrm{B}$ with trees $\mathrm{A}$ and $\mathrm{B}$ being subject and object, respectively + $\sum$ CMS over trees $A$ and $B$ with trees $A$ and $B$ as objective and subjective, respectively $) /(2 \mathrm{x}$ $\sum$ Number of branches over trees A and B ),

$$
\text { where } 0 \leq V_{\mathrm{c}} \leq 1 \text { and }
$$

$V_{c}^{\prime}: V_{c}$ obtained after the coarse-graining of one partner of a pair of trees which is more finely structured. This can be done by bunching level different clusters under a bunching criterion such as compression of less than $15 \%$ height difference.

\section{Case studies}

\subsection{Example 1 (pair of trees $A$ and $B$ ):}

\section{Case $\pi$ of Tree A as subject one;}

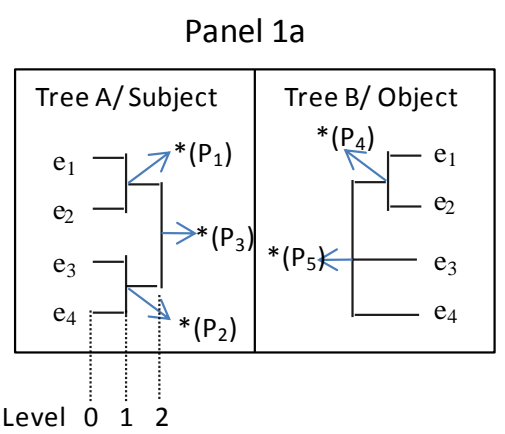

Panel $1 b$

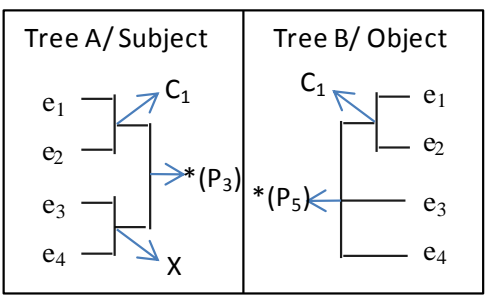

Panel 1c

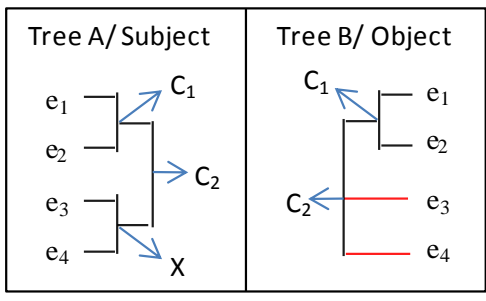

In Panel 1a, all of the possible clusters to be named are indicated with the star symbol (*).

First of all, the most basic level clusters of the subject tree $\mathrm{A}$ (i.e., $\mathrm{P}_{1}$ and $\mathrm{P}_{2}$ ) are subjected to the cluster matching examination against the clusters of the objective tree $\mathrm{B}$. Since the possible clusters, $\mathrm{P}_{1}$ and $\mathrm{P}_{4}$, contain exactly the same elements $\left(\mathrm{e}_{1}\right.$ and $\left.\mathrm{e}_{2}\right)$, they are assigned to be the congruent clusters and named as $\mathrm{C}_{1}$ (Panel 1b). On the contrary, $\mathrm{P}_{2}$ in the subject tree $\mathrm{A}$ cannot find a corresponding cluster in the objective tree $\mathrm{B}$ and thus cannot be named (denoted as $\mathrm{X}$ in Panel 1b). Now, we can see the trees with clusters named as in Panel 1b. Next level 2 
cluster is dealt: i.e., $\mathrm{P}_{3}$, which has the elements of $\mathrm{e}_{3}, \mathrm{e}_{4}$ and $\mathrm{C}_{1}$ (cluster-element). When the possible cluster $\mathrm{P}_{3}$ is compared with the clusters in the objective tree $\mathrm{B}, \mathrm{P}_{5}$ is an only possible candidate for the congruence cluster. Since the $\mathrm{P}_{5}$ cluster has the same elements, i.e., $\mathrm{e}_{3}, \mathrm{e}_{4}$ and $\mathrm{C}_{1}$, both $\mathrm{P}_{3}$ and $\mathrm{P}_{5}$ can be named with the same name, $\mathrm{C}_{2}$, as shown in Panel 1c.

\section{Case (- $\pi$ ) of Tree B as subject one:}

Panel 1d

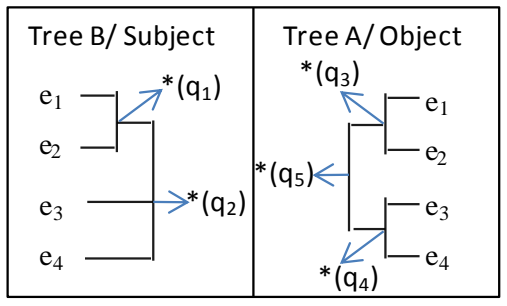

Panel 1e

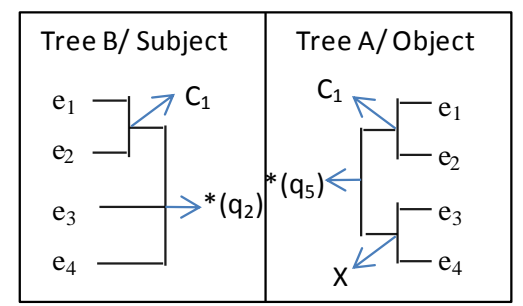

Panel 1f

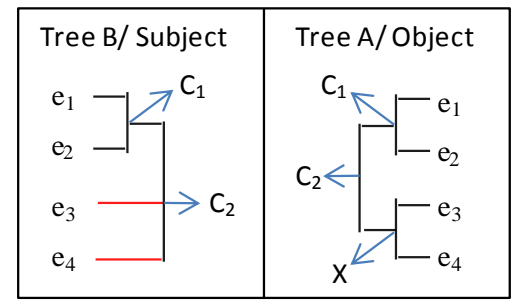

In the second session, the relationship of the subject and object is inversed for Trees A and B, rendering Tree A object and Tree B subject as in Panel 1d. Now, there are two $\left(q_{1}\right.$ and $\left.q_{2}\right)$ and three $\left(q_{3} \sim q_{5}\right)$ possible clusters in each tree.

Now, the most basic level cluster in the subjective tree $B$ is $q_{1}$ only. The $\mathrm{q}_{1}$ is examined to find its corresponding cluster in the objective tree A, providing with the corresponding one, i.e., $\mathrm{q}_{3}$ and thus assigned as $\mathrm{C}_{1}$ for both clusters.

Next, the higher level cluster $\mathrm{q}_{2}$ (here, it is the top level cluster) is subjected to the same examination using Panel 1e. The cluster $\mathrm{q}_{2}$ can find the corresponding matching cluster of $\mathrm{q}_{5}$ which also contains the same elements of $\mathrm{e}_{3}, \mathrm{e}_{4}$ and $\mathrm{C}_{1}$ as $\mathrm{q}_{2}$ cluster does. Therefore, $\mathrm{q}_{2}$ and $\mathrm{q}_{5}$ can be named with the same name, $\mathrm{C}_{2}$, as shown in Panel 1f. Let's count $V_{\mathrm{c}}$ for all of the possible pairs.

\section{CMS and the number of branches for the pair of Trees $A$ and B:}

For Case $\pi$ (Panel 1c)

\begin{tabular}{|c|c|c|c|c|}
\hline Tree name & Assigned cluster & Elements & CMS (Eq. adopted) & No. of branches \\
\hline \multirow{2}{*}{ Tree A (subject) } & $\mathrm{C}_{1}$ & $\mathrm{e}_{1}, \mathrm{e}_{2}$ & 2 (Eq. 1) & \multirow{2}{*}{$\begin{array}{c}(1+1+1+1+1+1) \\
=6\end{array}$} \\
\hline & $\mathrm{C}_{2}$ & $\mathrm{C}_{1}, \mathrm{e}_{3}, \mathrm{e}_{4}$ & $(1+.5+.5)=2($ Eq. 2$)$ & \\
\hline \multirow{2}{*}{ Tree B (object) } & $\mathrm{C}_{1}$ & $\mathrm{e}_{1}, \mathrm{e}_{2}$ & 2 (Eq. 1) & \multirow{2}{*}{$\begin{array}{c}(1+1+1+.5+.5) \\
=4\end{array}$} \\
\hline & $\mathrm{C}_{2}$ & $\mathrm{C}_{1}, \mathrm{e}_{3}, \mathrm{e}_{4}$ & 2 (Eq. 2) & \\
\hline \multicolumn{3}{|l|}{ Total } & 8 & 10 \\
\hline
\end{tabular}

For Case $-\pi$ (Panel 1f)

\begin{tabular}{|c|c|c|c|c|}
\hline Tree name & Assigned cluster & Elements & CMS (Eq. adopted) & No. of branches \\
\hline \multirow{2}{*}{ Tree B (subject) } & $\mathrm{C}_{1}$ & $\mathrm{e}_{1}, \mathrm{e}_{2}$ & 2 (Eq. 1) & $(1+1+1+.5+.5)$ \\
\hline & $\mathrm{C}_{2}$ & $\mathrm{C}_{1}, \mathrm{e}_{3}, \mathrm{e}_{4}$ & $(1+.5+.5)=2$ (Eq. 2$)$ & $=4$ \\
\hline \multirow{2}{*}{ Tree A (object) } & $\mathrm{C}_{1}$ & $\mathrm{e}_{1}, \mathrm{e}_{2}$ & 2 (Eq. 1) & $(1+1+1+1+1+1)$ \\
\hline & $\mathrm{C}_{2}$ & $\mathrm{C}_{1}, \mathrm{e}_{3}, \mathrm{e}_{4}$ & 2 (Eq. 2) & $=6$ \\
\hline \multicolumn{3}{|l|}{ Total } & 8 & 10 \\
\hline
\end{tabular}

Congruence value $\left(V_{c}\right)$ :

$$
V_{c}=(8+8) /(2 \times 10)=16 / 20=0.8
$$




\subsection{Example 2 (pair of Tree $C$ and Tree $D$ ):}

\section{Case $\pi$ of Tree $C$ as subject one;}

Panel 2a

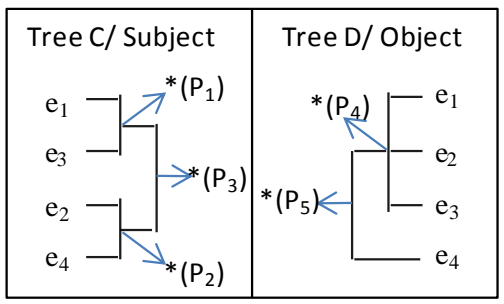

Panel 2b

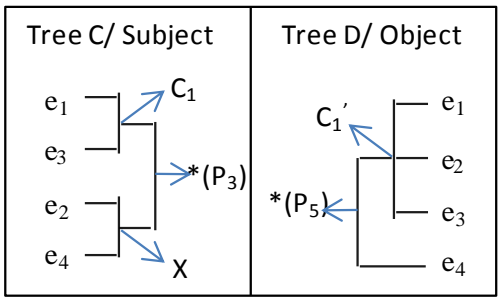

Panel 2c

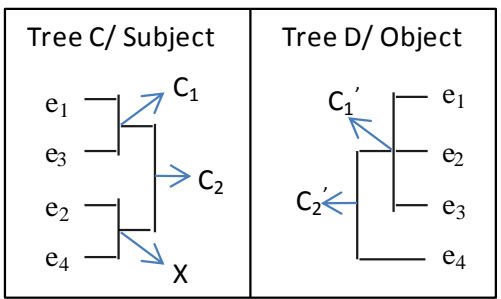

In Panel $2 \mathrm{a}$, there are three $\left(\mathrm{P}_{1} \sim \mathrm{P}_{3}\right)$ and two $\left(\mathrm{P}_{4} \sim \mathrm{P}_{5}\right)$ possible corresponding clusters in this case.

In the first step, the most basic level clusters of the subject tree $\mathrm{C}$ (i.e., $\mathrm{P}_{1}$ and $\mathrm{P}_{2}$ ) are subjected to the cluster matching examination against the clusters of the objective tree $\mathrm{D}$. $\mathrm{P}_{1}$ of the subject tree contains two elements, $\mathrm{e}_{1}$ and $\mathrm{e}_{3}$, and $\mathrm{P}_{4}$ of the object tree also contains $\mathrm{e}_{1}$ and $\mathrm{e}_{3}$ along with $\mathrm{e}_{2}$ at the same cluster level. Therefore, $\mathrm{P}_{1} \& \mathrm{P}_{4}$ can be assigned as $\mathrm{C}_{1}$ and $\mathrm{C}_{1}$ ' based on criteria 2, respectively (Panel $2 \mathrm{~b}$ ). On the contrary, $\mathrm{P}_{2}$ in the subject tree $\mathrm{C}$ cannot find a corresponding cluster in the objective tree D and thus cannot be named (denoted as X (extra naming) in Panel 2b).

The second step deals with the higher level cluster of subjective tree $\mathrm{C}$, i.e., $\mathrm{P}_{3}$, which has the elements of $\mathrm{e}_{2}, \mathrm{e}_{4}$ and $\mathrm{C}_{1}$ (cluster-element). When the cluster $\mathrm{P}_{3}$ is compared with the clusters in the objective tree $\mathrm{D}, \mathrm{P}_{5}$ is an only possible candidate for the congruence cluster. $\mathrm{P}_{5}$ cluster is composed of elements, $\mathrm{e}_{2}, \mathrm{e}_{4}$ and $\mathrm{C}_{1}$ ' and thus based on criteria 2, $\mathrm{P}_{3}$ and $\mathrm{P}_{5}$ can be named with the name $\mathrm{C}_{2}$ and $\mathrm{C}_{2}$, , respectively as shown in Panel 2c.

\section{Case $(-\pi)$ of Tree $D$ as subject one;}

Panel 2d

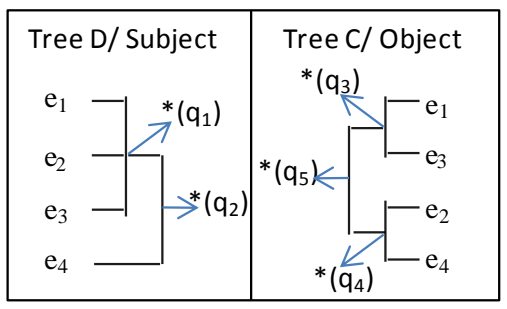

Panel 2e

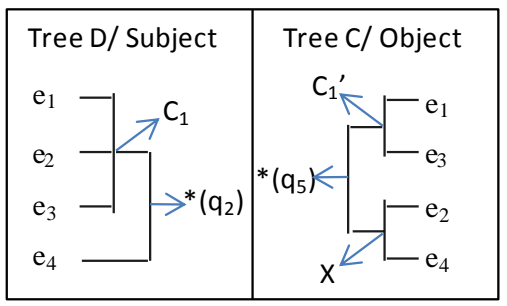

Panel $2 f$

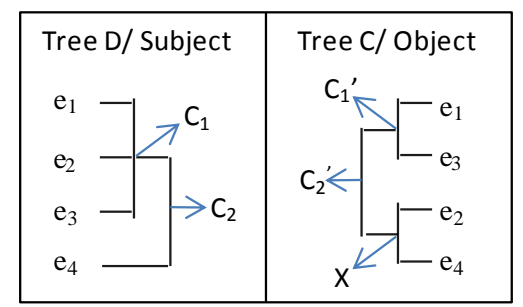

In the second session, the relationship of the subject and object is inversed, rendering the tree $\mathrm{C}$ object and the tree D subject as in Panel $2 \mathrm{~d}$. Now, there are two $\left(\mathrm{q}_{1}\right.$ and $\left.\mathrm{q}_{2}\right)$ and three $\left(\mathrm{q}_{3} \sim \mathrm{q}_{5}\right)$ possible clusters in each tree, respectively.

Then, the most basic level cluster in the subjective tree $D$ is $q_{1}$ only, containing three elements, $\mathrm{e}_{1}, \mathrm{e}_{2}$ and $\mathrm{e}_{3}$. The cluster $\mathrm{q}_{1}$ is examined to find its corresponding cluster in the objective tree $\mathrm{C}$ and can find $\mathrm{q}_{3}$ (containing two elements, $\mathrm{e}_{1}$ and $\mathrm{e}_{3}$ ), which is more than $50 \%$ of 
the number of $\mathrm{q}_{1}$ elements (three). Therefore, based on the assigning criteria $2, \mathrm{q}_{1}$ and $\mathrm{q}_{3}$ can be assigned as $\mathrm{C}_{1}$ and $\mathrm{C}_{1}$, , respectively (Panel 2e).

Next, the higher level cluster $\mathrm{q}_{2}$ is subjected to the same examination using Panel 2e. The cluster $\mathrm{q}_{2}$ (having the elements of $\mathrm{C}_{1}$ and $\mathrm{e}_{4}$ ) has a chance for finding the corresponding matching cluster of $\mathrm{q}_{5}$ which contains the elements of $\mathrm{e}_{2}, \mathrm{e}_{4}$ and $\mathrm{C}_{1}$ '. In this case, $\mathrm{e}_{4}$ representing as a common element for both trees and $\mathrm{C}_{1}$ ' of object tree representing the more than $50 \%$ of $\mathrm{C}_{1}$ cluster elements. Therefore, as a whole $\mathrm{q}_{5}$ cluster has $>50 \%$ elements of $\mathrm{q}_{2}$ cluster of the subject tree and thus $\mathrm{q}_{2}$ and $\mathrm{q}_{5}$ can be assigned as $\mathrm{C}_{2}$ and $\mathrm{C}_{2}$, , respectively (Panel 2f).

\section{CMS and the number of branches for the pair of Trees $C$ and D:}

For Case $\pi$ (Panel 2c)

\begin{tabular}{|c|c|c|c|c|}
\hline Tree name & Assigned cluster & Elements & CMS (Eq. adopted) & No. of branches \\
\hline \multirow{2}{*}{ Tree C (subject) } & $\mathrm{C}_{1}$ & $\mathrm{e}_{1}, \mathrm{e}_{3}$ & 2 (Eq. 1) & \multirow{2}{*}{$\begin{array}{c}(1+1+1+1+1+1) \\
=6\end{array}$} \\
\hline & $\mathrm{C}_{2}$ & $\mathrm{C}_{1}, \mathrm{e}_{2}, \mathrm{e}_{4}$ & $(1+.5+.5)=2$ (Eq. 2$)$ & \\
\hline \multirow[t]{2}{*}{ Tree D (object) } & $\mathrm{C}_{1}{ }^{\prime}$ & $\begin{array}{l}2\left(\mathrm{e}_{1}, \mathrm{e}_{3}\right) \text { of } \\
3\left(\mathrm{e}_{1}, \mathrm{e}_{2}, \mathrm{e}_{3}\right)\end{array}$ & 2/3 (Eq. 3) & \multirow{2}{*}{$\begin{array}{c}(1+1+1+1+.5) \\
=4.5\end{array}$} \\
\hline & $\mathrm{C}_{2}{ }^{\prime}$ & $\mathrm{C}_{1}^{\prime}, \mathrm{e}_{2}, \mathrm{e}_{4}$ & $1+.5+.5=2$ (Eq. 4$)$ & \\
\hline \multicolumn{3}{|l|}{ Total } & 6.66 & 10.5 \\
\hline
\end{tabular}

For Case $(-\pi)$ (Panel 2f)

\begin{tabular}{|c|c|c|c|c|}
\hline Tree name & Assigned cluster & Elements & CMS (Eq. adopted) & No. of branches \\
\hline \multirow{2}{*}{ Tree D (subject) } & $\mathrm{C}_{1}$ & $\mathrm{e}_{1}, \mathrm{e}_{2}, \mathrm{e}_{3}$ & 3 (Eq. 1) & $(1+1+1+1+.5)$ \\
& $\mathrm{C}_{2}$ & $\mathrm{C}_{1}, \mathrm{e}_{4}$ & $(1+.5)=1.5$ (Eq. 2) & $=4.5$ \\
\hline \multirow{3}{*}{ Tree C (object) } & $\mathrm{C}_{1}{ }^{\prime}$ & $\begin{array}{c}2\left(\mathrm{e}_{1}, \mathrm{e}_{3}\right) \text { of } \\
3\left(\mathrm{e}_{1}, \mathrm{e}_{2}, \mathrm{e}_{3}\right)\end{array}$ & 0.66 (Eq. 3) & \multirow{2}{*}{$(1+1+1+1+1+1)$} \\
& & $\mathrm{C}_{1}, \mathrm{e}_{2}, \mathrm{e}_{4}$ & $1+.5+.5=2$ (Eq. 2) & $=6$ \\
\cline { 2 - 4 } & $\mathrm{C}_{2}{ }^{\prime}$ & $\mathbf{7 . 1 6}$ & $\mathbf{1 0 . 5}$ \\
\hline \multicolumn{4}{|l}{ Total }
\end{tabular}

Congruence value $\left(V_{c}\right)$ :

$$
V_{\mathrm{c}}=(6.66+7.16) /(2 \times 10.5)=13.82 / 20.5=0.65
$$

\subsection{Example 3 (pair of Tree $\mathbf{E}$ and Tree $F$ ):}

Case $\pi$ of Tree $E$ as subject one;

Panel 3a

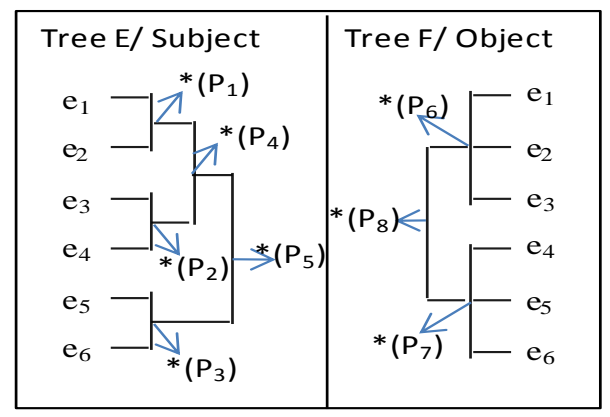

Panel 3b

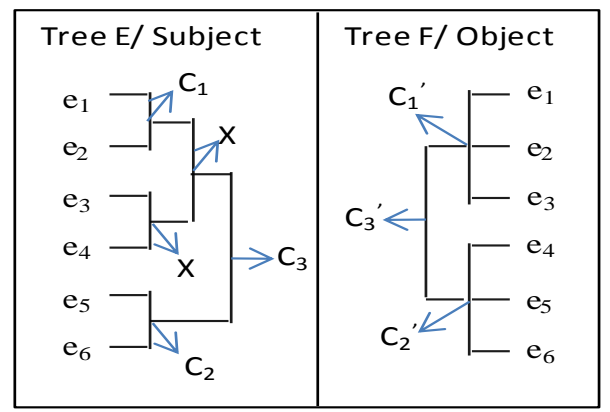


In panel 3a, there are five $\left(\mathrm{P}_{1} \sim \mathrm{P}_{5}\right)$ and three $\left(\mathrm{P}_{6} \sim \mathrm{P}_{8}\right)$ possible corresponding clusters in this case. Following the same algorithm as adopted in Cases 1 and 2, clusters $\mathrm{C}_{1} \sim \mathrm{C}_{3}$ and $\mathrm{C}_{1}{ }^{\prime}, \mathrm{C}_{2}$ ' and $\mathrm{C}_{3}$ ' can be assigned as shown in Panel $3 b$.

\section{Case $(-\pi)$ of Tree $F$ as subject one;}

Panel 3c

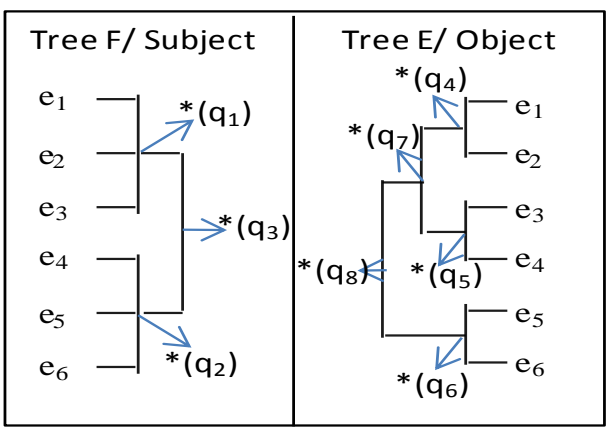

Panel3d

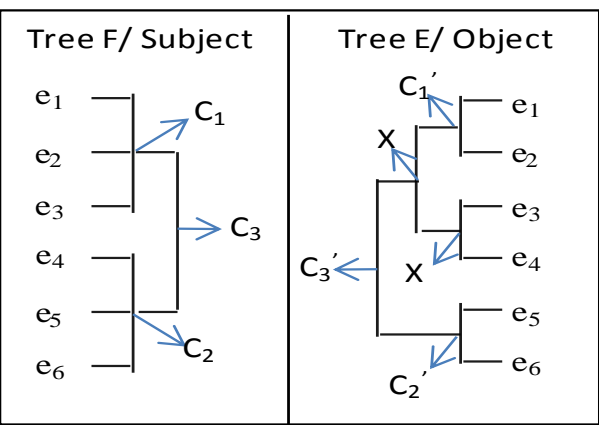

In the second session where the relationship of the subject and object is inversed, the named clusters $\mathrm{C}_{1} \sim \mathrm{C}_{3}$ and $\mathrm{C}_{1}{ }^{\prime}, \mathrm{C}_{2}{ }^{\prime}$ and $\mathrm{C}_{3}$ ' can be assigned as shown in Panel $3 \mathrm{~d}$.

\section{CMS and the number of branches for the pair of Trees $\mathrm{E}$ and $\mathrm{F}$ :}

For Case $\pi$ (Panel 3b)

\begin{tabular}{|c|c|c|c|c|}
\hline Tree name & Assigned cluster & Elements & CMS (Eq. adopted) & No. of branches \\
\hline \multirow{3}{*}{$\begin{array}{c}\text { Tree E } \\
\text { (subject) }\end{array}$} & $\mathrm{C}_{1}$ & $\mathrm{e}_{1}, \mathrm{e}_{2}$ & 2 (Eq. 1) & \multirow{3}{*}{$\begin{array}{c}(1+1+1+1+1+1+1+1 \\
+1+.5) \\
=9.5\end{array}$} \\
\hline & $\mathrm{C}_{2}$ & $\mathrm{e}_{5}, \mathrm{e}_{6}$ & 2 (Eq. 1) & \\
\hline & $\mathrm{C}_{3}$ & $\mathrm{C}_{1}, \mathrm{e} 3, \mathrm{e}_{4}, \mathrm{C}_{2}$ & $.5+.25+.25+.5=1.5 \quad$ (Eq. 2) & \\
\hline \multirow{3}{*}{$\begin{array}{l}\text { Tree F } \\
\text { (object) }\end{array}$} & $\mathrm{C}_{1}{ }^{\prime}$ & $2\left(e_{1}, e_{2}\right)$ of $3\left(e_{1}, e_{2}, e_{3}\right)$ & 2/3 (Eq. 3) & \multirow{3}{*}{$\begin{array}{c}(1+1+1+1+1+1+1 \\
+1) \\
=8\end{array}$} \\
\hline & $\mathrm{C}_{2}{ }^{\prime}$ & $2\left(\mathrm{e}_{5}, \mathrm{e}_{6}\right)$ of $3\left(\mathrm{e}_{4}, \mathrm{e}_{5}, \mathrm{e}_{6}\right)$ & 2/3 (Eq. 3) & \\
\hline & $\mathrm{C}_{3}{ }^{\prime}$ & $\mathrm{C}_{1}{ }^{\prime}, \mathrm{e}_{3}, \mathrm{e}_{4}, \mathrm{C}_{2}{ }^{\prime}$ & $1+.5+.5+1=2.5$ (Eq. 4$)$ & \\
\hline \multicolumn{3}{|l|}{ Total } & 9.32 & 17.5 \\
\hline
\end{tabular}

For Case (- $\pi$ ) (Panel 3d)

\begin{tabular}{|c|c|c|c|c|}
\hline Tree name & Assigned cluster & Elements & CMS (Eq. adopted) & No. of branches \\
\hline \multirow{3}{*}{$\begin{array}{c}\text { Tree F } \\
\text { (subject) }\end{array}$} & $\mathrm{C}_{1}$ & $\mathrm{e}_{1}, \mathrm{e}_{2}, \mathrm{e}_{3}$ & 3 (Eq. 1) & \multirow{3}{*}{$\begin{array}{c}(1+1+1+1+1+1+1 \\
+1) \\
=8\end{array}$} \\
\hline & $\mathrm{C}_{2}$ & $\mathrm{e}_{4}, \mathrm{e}_{5}, \mathrm{e}_{6}$ & 3 (Eq. 1) & \\
\hline & $\mathrm{C}_{3}$ & $\mathrm{C}_{1}, \mathrm{C}_{2}$ & $1+1=2 \quad$ (Eq. 2) & \\
\hline \multirow{3}{*}{$\begin{array}{c}\text { Tree E } \\
\text { (object) }\end{array}$} & $\mathrm{C}_{1}{ }^{\prime}$ & $2\left(e_{1}, e_{2}\right)$ of $3\left(e_{1}, e_{2}, e_{3}\right)$ & 2/3 (Eq. 3) & \multirow{3}{*}{$\begin{array}{c}(1+1+1+1+1+1+1+1 \\
+1+.5) \\
=9.5\end{array}$} \\
\hline & $\mathrm{C}_{2}{ }^{\prime}$ & $2\left(e_{5}, e_{6}\right)$ of $3\left(e_{4}, e_{5}, e_{6}\right)$ & 2/3 (Eq. 3) & \\
\hline & $\mathrm{C}_{3}{ }^{\prime}$ & $\mathrm{C}_{1}{ }^{\prime}, \mathrm{e}_{3}, \mathrm{e}_{4}, \mathrm{C}_{2}{ }^{\prime}$ & $.5+.25+.25+.5=1.5$ (Eq. 4$)$ & \\
\hline \multicolumn{3}{|l|}{ Total } & 10.82 & $\mathbf{1 7 . 5}$ \\
\hline
\end{tabular}

\section{Congruence value $\left(V_{c}\right)$ :}

$$
V_{c}=(9.32+10.82) /(2 \times 17.5)=20.14 / 35=0.57
$$




\subsection{Example 4 (pair of Tree $\mathbf{G}$ and Tree $H$ ):}

\section{Case $\pi$ of Tree $G$ as subject one;}

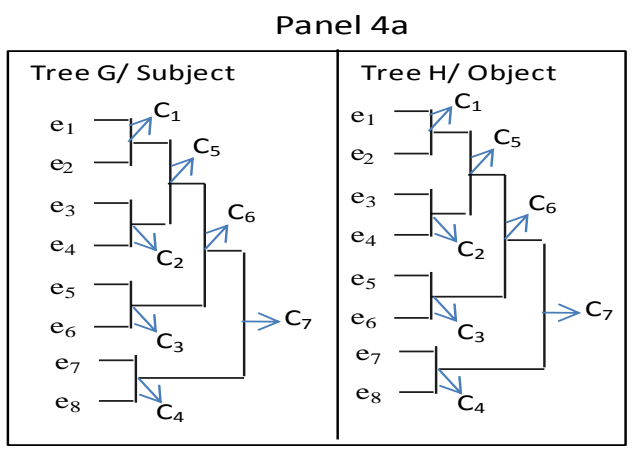

Case $(-\pi)$ of Tree $H$ as subject one;

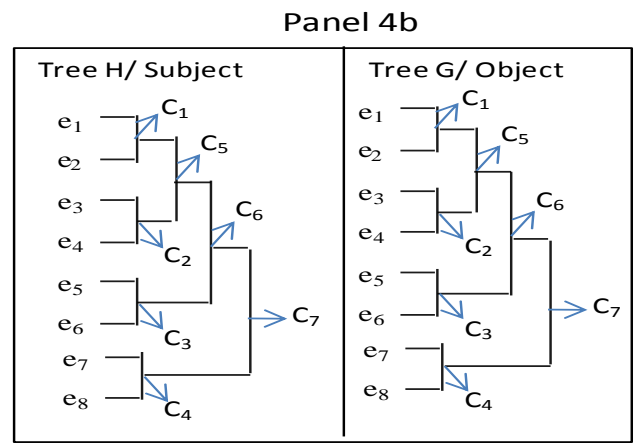

CMS and the number of branches for the pair of Trees $G$ and $H$ :

For Case $\pi$ (Panel 4a)

\begin{tabular}{|c|c|c|c|c|}
\hline Tree name & Assigned cluster & Elements & CMS (Eq. adopted) & No. of branches \\
\hline \multirow{7}{*}{$\begin{array}{c}\text { Tree G } \\
\text { (subject) }\end{array}$} & $\mathrm{C}_{1}$ & $\mathrm{e}_{1}, \mathrm{e}_{2}$ & 2 (Eq. 1) & \multirow{7}{*}{$\begin{array}{c}(1+1+1+1+1+1+1+1+1 \\
+1+1+1+.5+.25) \\
=12.75\end{array}$} \\
\hline & $\mathrm{C}_{2}$ & $\mathrm{e}_{3}, \mathrm{e}_{4}$ & 2 (Eq. 1) & \\
\hline & $\mathrm{C}_{3}$ & $\mathrm{e}_{5}, \mathrm{e}_{6}$ & 2 (Eq. 1) & \\
\hline & $\mathrm{C}_{4}$ & $\mathrm{e}_{7}, \mathrm{e}_{8}$ & 2 (Eq. 1) & \\
\hline & $\mathrm{C}_{5}$ & $\mathrm{C}_{1}, \mathrm{C}_{2}$ & 2 (Eq. 2) & \\
\hline & $\mathrm{C}_{6}$ & $\mathrm{C}_{5}, \mathrm{C}_{3}$ & $1+.5=1.5$ (Eq. 2) & \\
\hline & $\mathrm{C}_{7}$ & $\mathrm{C}_{6}, \mathrm{C}_{4}$ & $1+.25=1.25$ (Eq. 2$)$ & \\
\hline \multirow{7}{*}{$\begin{array}{c}\text { Tree H } \\
\text { (object) }\end{array}$} & $\mathrm{C}_{1}$ & $\mathrm{e}_{1}, \mathrm{e}_{2}$ & 2 (Eq. 1) & \multirow{7}{*}{$\begin{array}{c}(1+1+1+1+1+1+1+1+1 \\
+1+1+1+.5+.25) \\
=12.75\end{array}$} \\
\hline & $\mathrm{C}_{2}$ & $\mathrm{e}_{3}, \mathrm{e}_{4}$ & 2 (Eq. 1) & \\
\hline & $\mathrm{C}_{3}$ & $\mathrm{e}_{5}, \mathrm{e}_{6}$ & 2 (Eq. 1) & \\
\hline & $\mathrm{C}_{4}$ & $\mathrm{e}_{7,}, \mathrm{e}_{8}$ & 2 (Eq. 1) & \\
\hline & $\mathrm{C}_{5}$ & $\mathrm{C}_{1}, \mathrm{C}_{2}$ & 2 (Eq. 2) & \\
\hline & $\mathrm{C}_{6}$ & $\mathrm{C}_{5}, \mathrm{C}_{3}$ & $1+.5=1.5$ (Eq. 2) & \\
\hline & $\mathrm{C}_{7}$ & $\mathrm{C}_{6}, \mathrm{C}_{4}$ & $1+.25=1.25$ (Eq. 2$)$ & \\
\hline \multicolumn{3}{|l|}{ Total } & 25.5 & 25.5 \\
\hline
\end{tabular}

For Case (- $\pi$ ) (Panel 4b)

\begin{tabular}{|c|c|c|c|c|}
\hline Tree name & Assigned cluster & Elements & CMS (Eq. adopted) & No. of branches \\
\hline \multirow{7}{*}{$\begin{array}{c}\text { Tree H } \\
\text { (subject) }\end{array}$} & $\mathrm{C}_{1}$ & $\mathrm{e}_{1}, \mathrm{e}_{2}$ & 2 (Eq. 1) & \multirow{7}{*}{$\begin{array}{c}(1+1+1+1+1+1+1+1+1 \\
+1+1+1+.5+.25) \\
=12.75\end{array}$} \\
\hline & $\mathrm{C}_{2}$ & $e_{3,} e_{4}$ & 2 (Eq. 1) & \\
\hline & $\mathrm{C}_{3}$ & $\mathrm{e}_{5,} \mathrm{e}_{6}$ & 2 (Eq. 1) & \\
\hline & $\mathrm{C}_{4}$ & $e_{7}, e_{8}$ & 2 (Eq. 1) & \\
\hline & $\mathrm{C}_{5}$ & $\mathrm{C}_{1}, \mathrm{C}_{2}$ & 2 (Eq. 2) & \\
\hline & $\mathrm{C}_{6}$ & $\mathrm{C}_{5}, \mathrm{C}_{3}$ & $1+.5=1.5$ (Eq. 2 ) & \\
\hline & $\mathrm{C}_{7}$ & $\mathrm{C}_{6}, \mathrm{C}_{4}$ & $1+.25=1.25$ (Eq. 2$)$ & \\
\hline \multirow{7}{*}{$\begin{array}{l}\text { Tree G } \\
\text { (object) }\end{array}$} & $\mathrm{C}_{1}$ & $\mathrm{e}_{1}, \mathrm{e}_{2}$ & 2 (Eq. 1) & \multirow{7}{*}{$\begin{array}{c}(1+1+1+1+1+1+1+1+1 \\
+1+1+1+.5+.25) \\
=12.75\end{array}$} \\
\hline & $\mathrm{C}_{2}$ & $\mathrm{e}_{3,}, \mathrm{e}_{4}$ & 2 (Eq. 1) & \\
\hline & $\mathrm{C}_{3}$ & $\mathrm{e}_{5}, \mathrm{e}_{6}$ & 2 (Eq. 1) & \\
\hline & $\mathrm{C}_{4}$ & $\mathrm{e}_{7}, \mathrm{e}_{8}$ & 2 (Eq. 1) & \\
\hline & $\mathrm{C}_{5}$ & $\mathrm{C}_{1}, \mathrm{C}_{2}$ & 2 (Eq. 2) & \\
\hline & $\mathrm{C}_{6}$ & $\mathrm{C}_{5}, \mathrm{C}_{3}$ & $1+.5=1.5$ (Eq. 2$)$ & \\
\hline & $\mathrm{C}_{7}$ & $\mathrm{C}_{6}, \mathrm{C}_{4}$ & $1+.25=1.25$ (Eq. 2$)$ & \\
\hline \multicolumn{3}{|l|}{ Total } & 25.5 & 25.5 \\
\hline
\end{tabular}

Congruence value $\left(V_{c}\right): V_{c}=(25.5+25.5) /(2 \times 25.5)=1$ 
3.5 Example 5 (pair of Tree I and Tree J):

\section{Case $\pi$ of Tree $I$ as subject one;}

Panel5a

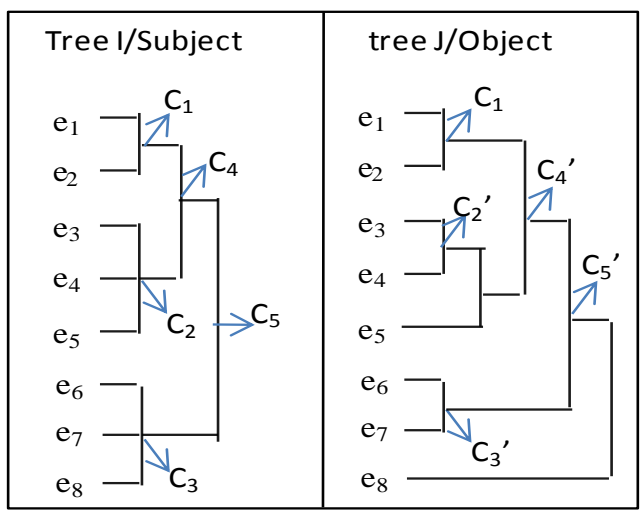

Case $(-\pi)$ of Tree $J$ as subject one;

Panel $5 b$

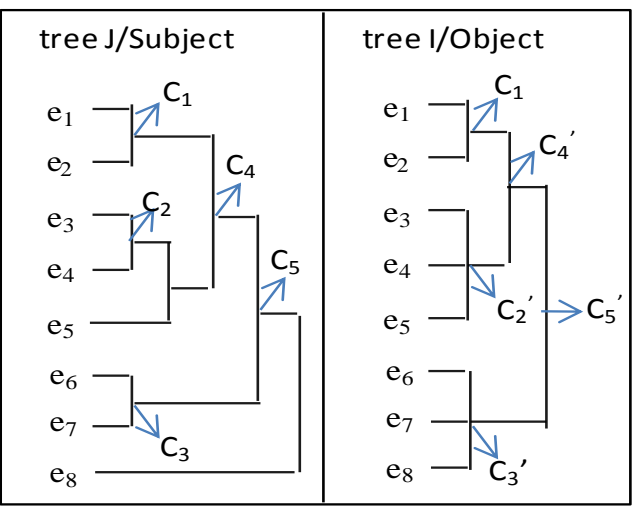

CMS and the number of branches for the pair of Trees I and J:

For Case $\pi$ (Panel 5a)

\begin{tabular}{|c|c|c|c|c|}
\hline Tree name & Assigned cluster & Elements & CMS (Eq. adopted) & No. of branches \\
\hline \multirow{5}{*}{$\begin{array}{c}\text { Tree I } \\
\text { (subject) }\end{array}$} & $\mathrm{C}_{1}$ & $\mathrm{e}_{1}, \mathrm{e}_{2}$ & 2 (Eq. 1) & \multirow{5}{*}{$\begin{array}{c}(1+1+1+1+1+1+1+1+ \\
1+1+1+.5) \\
=11.5\end{array}$} \\
\hline & $\mathrm{C}_{2}$ & $\mathrm{e}_{3}, \mathrm{e}_{4}, \mathrm{e}_{5}$ & 3 (Eq. 1) & \\
\hline & $\mathrm{C}_{3}$ & $\mathrm{e}_{6}, \mathrm{e}_{7}, \mathrm{e}_{8}$ & 3 (Eq. 1) & \\
\hline & $\mathrm{C}_{4}$ & $\mathrm{C}_{1}, \mathrm{C}_{2}$ & $1+1=2$ (Eq. 2 ) & \\
\hline & $\mathrm{C}_{5}$ & $\mathrm{C}_{4}, \mathrm{C}_{3}$ & $1+.5=1.5$ (Eq. 2$)$ & \\
\hline \multirow{5}{*}{$\begin{array}{c}\text { Tree J } \\
\text { (object) }\end{array}$} & $\mathrm{C}_{1}$ & $\mathrm{e}_{1}, \mathrm{e}_{2}$ & 2 (Eq. 1) & \multirow{5}{*}{$\begin{array}{c}(1+1+1+1+1+1+1+1+ \\
1+.5+.5+.25+.12) \\
=10.37\end{array}$} \\
\hline & $\mathrm{C}_{2}$ & $2\left(e_{3}, e_{4}\right)$ of $3\left(e_{3}, e_{4}, e_{5}\right)$ & 2/3 (Eq. 3) & \\
\hline & $\mathrm{C}_{3}$ & $2\left(e_{6}, e_{7}\right)$ of $3\left(e_{6}, e_{7}, e_{8}\right)$ & $2 / 3$ (Eq. 3) & \\
\hline & $\mathrm{C}_{4}^{\prime}$ & $\mathrm{C}_{1}, \mathrm{C}_{2}^{\prime}, \mathrm{e}_{5}$ & $.5+.5+.25=1.25$ (Eq. 4$)$ & \\
\hline & $\mathrm{C}_{5}$ & $\mathrm{C}_{4}, \mathrm{C}_{3}$ & $1+.25=1.25$ (Eq. 4$)$ & \\
\hline \multicolumn{3}{|l|}{ Total } & 17.32 & 21.87 \\
\hline
\end{tabular}

For Case (- $\pi$ ) (Panel 5b)

\begin{tabular}{|c|c|c|c|c|}
\hline Tree name & Assigned cluster & Elements & CMS (Eq. adopted) & No. of branches \\
\hline \multirow{4}{*}{$\begin{array}{c}\text { Tree J } \\
\text { (subject) }\end{array}$} & $\mathrm{C}_{1}$ & $\mathrm{e}_{1}, \mathrm{e}_{2}$ & 2 (Eq. 1) & \multirow{3}{*}{$=10.37$} \\
\cline { 2 - 4 } & $\mathrm{C}_{2}$ & $\mathrm{e}_{3,} \mathrm{e}_{4}$ & 2 (Eq. 1) & \\
\cline { 2 - 5 } & $\mathrm{C}_{3}$ & $\mathrm{e}_{6}, \mathrm{e}_{7}$ & 2 (Eq. 1) & \multirow{2}{*}{$=11.5$} \\
\cline { 2 - 5 } & $\mathrm{C}_{4}$ & $\mathrm{C}_{1}, \mathrm{C}_{2}, \mathrm{e}_{5}$ & $.5+.5+.25=1.25$ (Eq. 2) \\
\cline { 2 - 5 } $\begin{array}{c}\text { Tree I } \\
\text { (object) }\end{array}$ & $\mathrm{C}_{5}$ & $\mathrm{C}_{4}, \mathrm{C}_{3}$ & $1+.25=1.25$ (Eq. 2) & \\
\cline { 2 - 5 } & $\mathrm{C}_{1}$ & $\mathrm{e}_{1}, \mathrm{e}_{2}$ & 2 (Eq. 1) & \\
\cline { 2 - 5 } & $\mathrm{C}_{2}$ & $2\left(\mathrm{e}_{3}, \mathrm{e}_{4}\right)$ of $3\left(\mathrm{e}_{3}, \mathrm{e}_{4}, \mathrm{e}_{5}\right)$ & $2 / 3$ (Eq. 3) & \\
\cline { 2 - 5 } & $\mathrm{C}_{3}$ & $2\left(\mathrm{e}_{6}, \mathrm{e}_{7}\right)$ of $3\left(\mathrm{e}_{6}, \mathrm{e}_{7}, \mathrm{e}_{8}\right)$ & $2 / 3$ (Eq. 3) & $\mathbf{2 1 . 8 7}$ \\
\hline
\end{tabular}

Congruence value $\left(V_{c}\right)$ :

$$
V_{\mathrm{c}}=(17.32+15.32) /(2 \times 21.87)=32.64 / 43.74=0.74
$$




\subsection{Example 6 (pair of Tree $K$ and Tree $L$ ):}

\section{Case $\pi$ of Tree $\mathrm{K}$ as subject one;}

Panel 6a

\begin{tabular}{|l|l|}
\hline Tree K/ Subject & Tree L/Object \\
$\mathrm{e}_{1}-$ & $\mathrm{e}_{1}-$ \\
$\mathrm{e}_{2}-$ & $\mathrm{e}_{2}-$ \\
$\mathrm{e}_{3}-$ & $\mathrm{e}_{3}$ \\
$\mathrm{e}_{4}-$ & $\mathrm{e}_{4}$ \\
$\mathrm{e}_{5}-$ & $\mathrm{e}_{5}$ \\
\hline
\end{tabular}

Case $(-\pi)$ of Tree $L$ as subject one;

Panel 6b

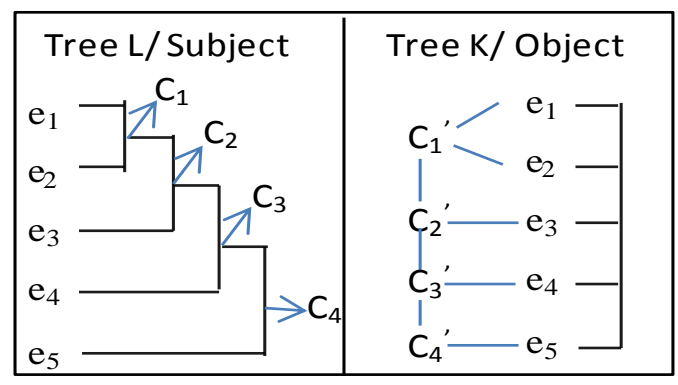

\section{CMS and the number of branches for the pair of Trees $K$ and $L$ :}

\section{For Case $\pi$ (Panel 6a)}

Based on criterion 3, no name is given as $\leq 50 \%$ elements of the subjective tree found the corresponding cluster contained in the objective tree.

\begin{tabular}{|c|c|c|c|c|}
\hline Tree name & Assigned cluster & Elements & CMS (Eq. adopted) & No. of branches \\
\hline \multirow{2}{*}{$\begin{array}{c}\text { Tree K } \\
\text { (subject) }\end{array}$} & & & & \multirow{2}{*}{$\begin{array}{c}(1+1+1+1+1) \\
=5\end{array}$} \\
\hline & & & & \\
\hline \multirow{2}{*}{$\begin{array}{c}\text { Tree L } \\
\text { (object) }\end{array}$} & & & & \multirow{2}{*}{$\begin{array}{c}(1+1+1+1+1+.5+.25+ \\
.12)=5.87\end{array}$} \\
\hline & & & & \\
\hline \multicolumn{2}{|l|}{ Total } & & 0.00 & 10.87 \\
\hline
\end{tabular}

For Case (- $\pi$ ) (Panel 6b)

\begin{tabular}{|c|c|c|c|c|}
\hline Tree name & Assigned cluster & Elements & CMS (Eq. adopted) & No. of branches \\
\hline \multirow{4}{*}{$\begin{array}{c}\text { Tree L } \\
\text { (subject) }\end{array}$} & $\mathrm{C}_{1}$ & $e_{1}, e_{2}$ & 2 (Eq. 1) & \multirow{4}{*}{$=5.87$} \\
\hline & $\mathrm{C}_{2}$ & $\mathrm{C}_{1}, \mathrm{e}_{3}$ & $1+.5=1.5$ (Eq. 2$)$ & \\
\hline & $\mathrm{C}_{3}$ & $\mathrm{C}_{2,} \mathrm{e}_{4}$ & $1+.25=1.25$ (Eq. 2$)$ & \\
\hline & $\mathrm{C}_{4}$ & $\mathrm{C}_{3}, \mathrm{e}_{5}$ & $1+.12=1.12$ (Eq. 2$)$ & \\
\hline \multirow{4}{*}{$\begin{array}{l}\text { Tree K } \\
\text { (object) }\end{array}$} & $\mathrm{C}_{1}^{\prime}$ & $2\left(\mathrm{e}_{1}, \mathrm{e}_{2}\right)$ of $5\left(\mathrm{e}_{1} \sim \mathrm{e}_{5}\right)$ & .005 (Eq. 3) & \multirow{4}{*}{$=5$} \\
\hline & $\mathrm{C}_{2}$ & $2\left(\mathrm{C}_{1}, \mathrm{e}_{3}\right)$ of 5 & .005 (Eq. 3) & \\
\hline & $\mathrm{C}_{3}$ & $2\left(\mathrm{C}_{2}, \mathrm{e}_{4}\right)$ of 5 & .005 (Eq. 3) & \\
\hline & $\overrightarrow{\mathrm{C}_{4}}$ & $2\left(\mathrm{C}_{3}, \mathrm{e}_{5}\right)$ of 5 & .005 (Eq. 3) & \\
\hline \multicolumn{3}{|l|}{ Total } & 5.89 & 10.87 \\
\hline
\end{tabular}

Congruence value $\left(V_{c}\right)$ :

$$
V_{c}=(0.00+5.89) /(2 \times 10.87)=5.89 / 21.74=0.27
$$




\section{Application to the current study}

\subsection{Congruence value $\left(V_{c}\right)$ between phenotype and $18 \mathrm{~S}$ rDNA-based (Fig. 2c) trees:}

Case $\pi$ of phenotype-based tree as subject one;

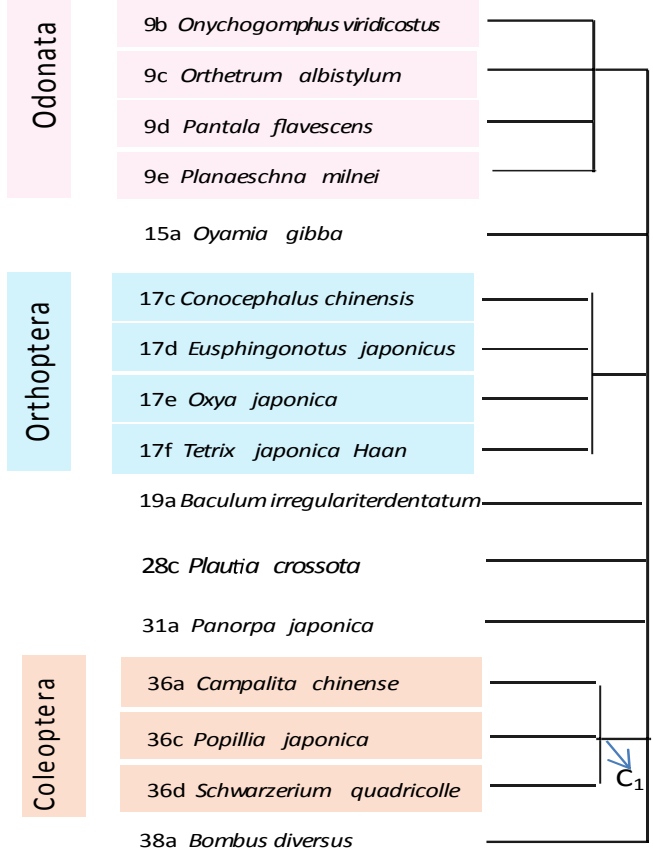

Phenotype-based/Subject tree

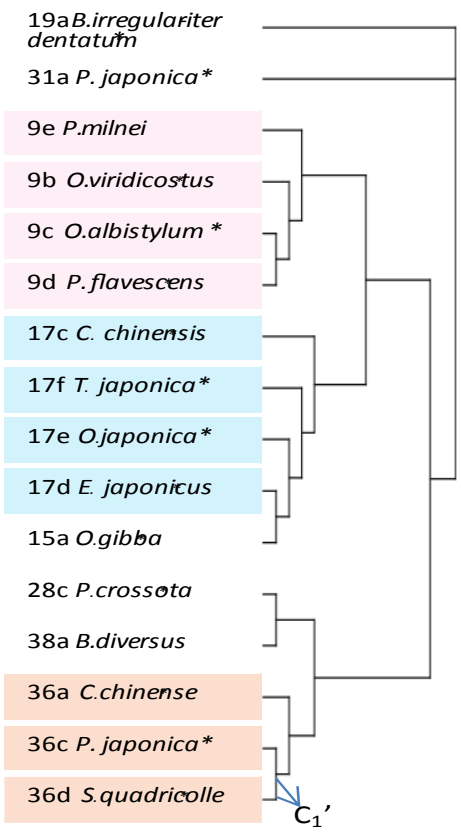

$18 S$ rDNA-based (Fig. 2c)/Object tree

Case $(-\pi)$ of $18 \mathrm{~S}$ rDNA-based as subject one;
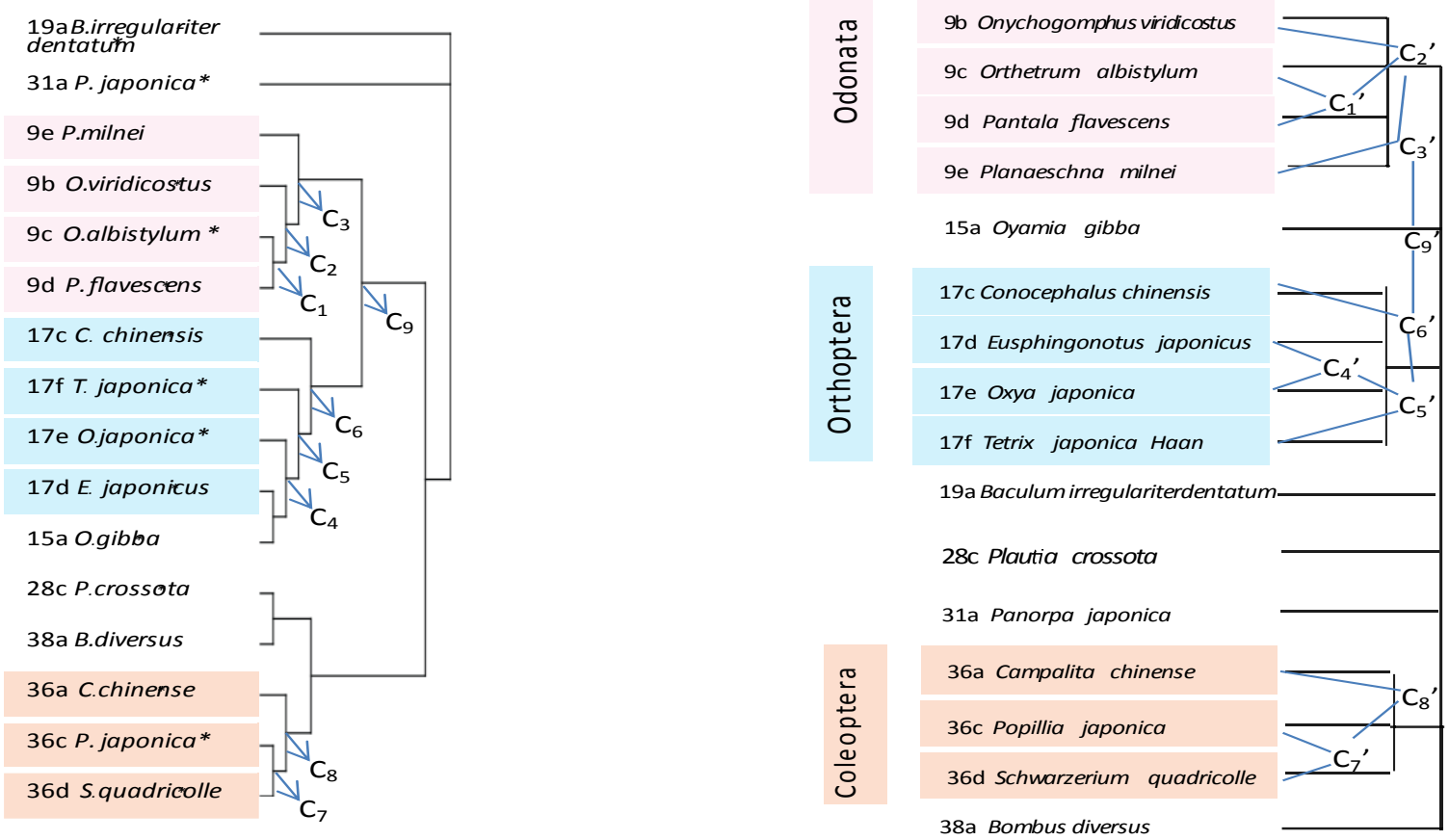

$18 S$ rDNA-based (Fig. 2c)/Subject tree
Phenotype-based/Object tree 
CMS and the number of branches for the pair of phenotype and 18S rDNA-based (Fig. 2c)

trees:

For Case $\pi$;

\begin{tabular}{|c|c|c|c|c|}
\hline Tree name & Assigned cluster & Elements & CMS (Eq. adopted) & No. of branches \\
\hline $\begin{array}{l}\text { Phenotype-based } \\
\text { tree (subject) }\end{array}$ & $\mathrm{C}_{1}$ & 36a, 36c, 36d & 3(Eq. 1) & $=16.5$ \\
\hline $\begin{array}{c}\text { 18S rDNA-based } \\
\text { tree (object) }\end{array}$ & $\mathrm{C}_{1}{ }^{\prime}$ & 2(36c, 36d) of 3 (36 series) & $2 / 3$ (Eq. 3) & $=17$ \\
\hline \multicolumn{2}{|l}{ Total } & & $\mathbf{3 . 6 6}$ & $\mathbf{3 3 . 5}$ \\
\hline
\end{tabular}

For Case $(-\pi)$;

\begin{tabular}{|c|c|c|c|c|}
\hline Tree name & Assigned cluster & Elements & CMS (Eq. adopted) & No. of branches \\
\hline \multirow{9}{*}{$\begin{array}{l}\text { 18S rDNA-based } \\
\text { tree (Subject) }\end{array}$} & $\mathrm{C}_{1}$ & $9 \mathrm{c}, 9 \mathrm{~d}$ & 2 (Eq. 1) & \multirow{9}{*}{$=17$} \\
\hline & $\mathrm{C}_{2}$ & $\mathrm{C}_{1}, 9 \mathrm{~b}$ & $1+.5=1.5$ (Eq. 2$)$ & \\
\hline & $\mathrm{C}_{3}$ & $\mathrm{C}_{4}, 9 \mathrm{e}$ & $1+.25=1.25 \quad$ (Eq. 2$)$ & \\
\hline & $\mathrm{C}_{4}$ & $17 \mathrm{e}, 17 \mathrm{~d}$ & $.5+.5=1$ (Eq. 2$)$ & \\
\hline & $\mathrm{C}_{5}$ & $\mathrm{C}_{4}, 17 \mathrm{f}$ & $1+.25=1.25$ (Eq. 2$)$ & \\
\hline & $\mathrm{C}_{6}$ & $\mathrm{C}_{5}, 17 \mathrm{c}$ & $1+.12=1.12$ (Eq. 2) & \\
\hline & $\mathrm{C}_{7}$ & $36 \mathrm{c}, 36 \mathrm{~d}$ & 2 (Eq. 1) & \\
\hline & $\mathrm{C}_{8}$ & $\mathrm{C}_{7}, 36 \mathrm{a}$ & $1+.5=1.5$ (Eq. 2$)$ & \\
\hline & $\mathrm{C}_{9}$ & $\mathrm{C}_{3}, \mathrm{C}_{6}$ & $.25+.12=.37$ (Eq. 2) & \\
\hline \multirow{9}{*}{$\begin{array}{l}\text { Phenotype-based } \\
\text { tree (Object) }\end{array}$} & $\mathrm{C}_{1}{ }^{\prime}$ & $2(9 c, 9 d)$ of $4(9$ series $)$ & 0.08 (Eq. 3) & \multirow{9}{*}{$=16.5$} \\
\hline & $\mathrm{C}_{2}^{\prime}$ & $9 \mathrm{~b}, \mathrm{C}_{1}{ }^{\prime}$ & 0.08 (Eq. 3) & \\
\hline & $\mathrm{C}_{3}$, & $9 \mathrm{e}, \mathrm{C}_{2}{ }^{\prime}$ & 0.08 (Eq. 3) & \\
\hline & $\mathrm{C}_{4}{ }^{\prime}$ & $17 \mathrm{~d}, 17 \mathrm{e}$ & 0.08 (Eq. 3) & \\
\hline & $\mathrm{C}_{5}^{\prime}$ & $17 \mathrm{f}, \mathrm{C}_{4}{ }^{\prime}$ & 0.08 (Eq. 3) & \\
\hline & $\mathrm{C}_{6}^{\prime}$ & $17 \mathrm{c}, \mathrm{C}_{5}{ }^{\prime}$ & 0.08 (Eq. 3) & \\
\hline & $\mathrm{C}_{7}^{\prime}$ & $36 c, 36 d$ & 2/3 (Eq. 3) & \\
\hline & $\mathrm{C}_{8}{ }^{\prime}$ & $36 \mathrm{a}, \mathrm{C}_{7}$ & 2/3 (Eq. 3) & \\
\hline & $\mathrm{C}_{9}{ }^{\prime}$ & 2 of $9(9 \& 17$ series $+15 a)$ & $<0.05$ (Eq. 3) & \\
\hline \multicolumn{3}{|l|}{ Total } & 13.79 & 33.5 \\
\hline
\end{tabular}

Congruence value $\left(V_{\mathrm{c}}\right)$ :

$$
V_{\mathrm{c}}=(3.66+13.79) /(2 \times 33.5)=17.45 / 67=0.26
$$




\subsection{Congruence value calculation between phenotype and 18S rDNA based (Fig. 2b)}

trees

\section{Case $\pi$ of phenotype-based tree as subject one;}

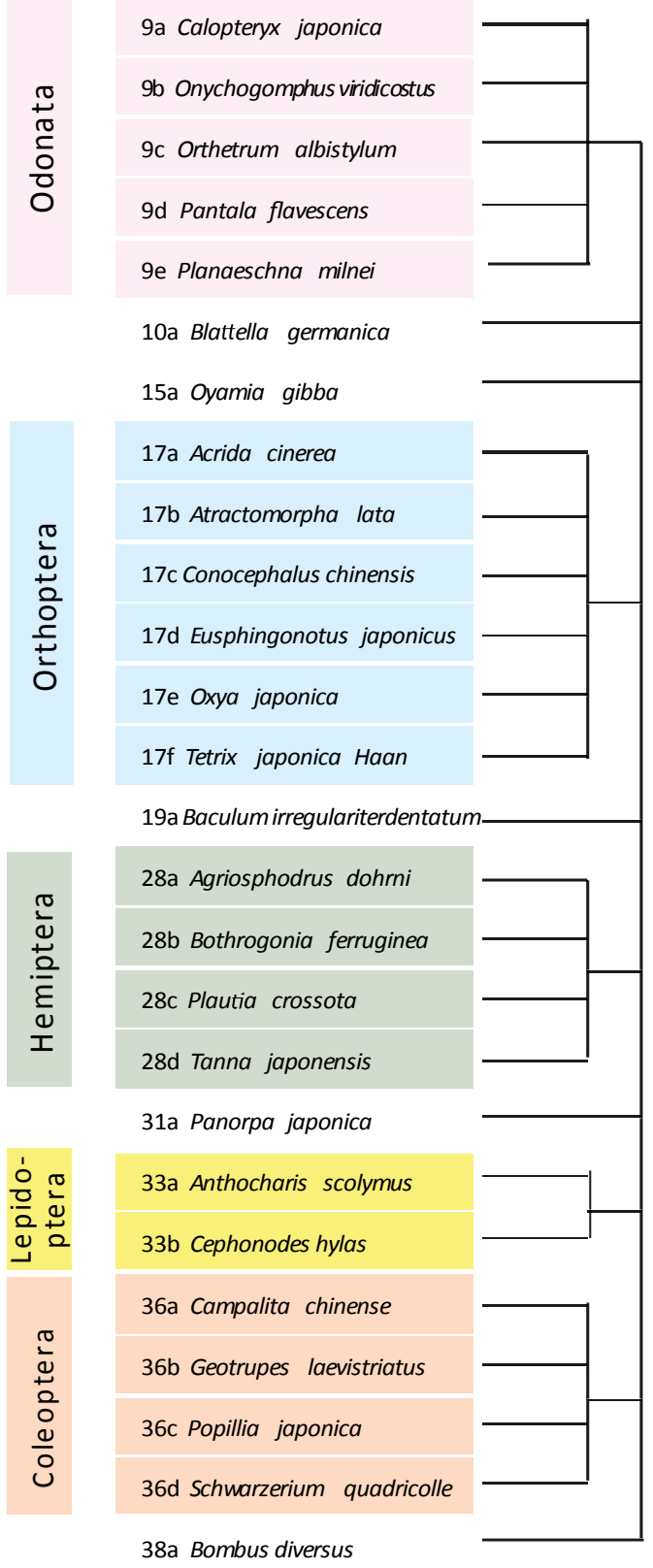

Phenotype-based/Subject tree

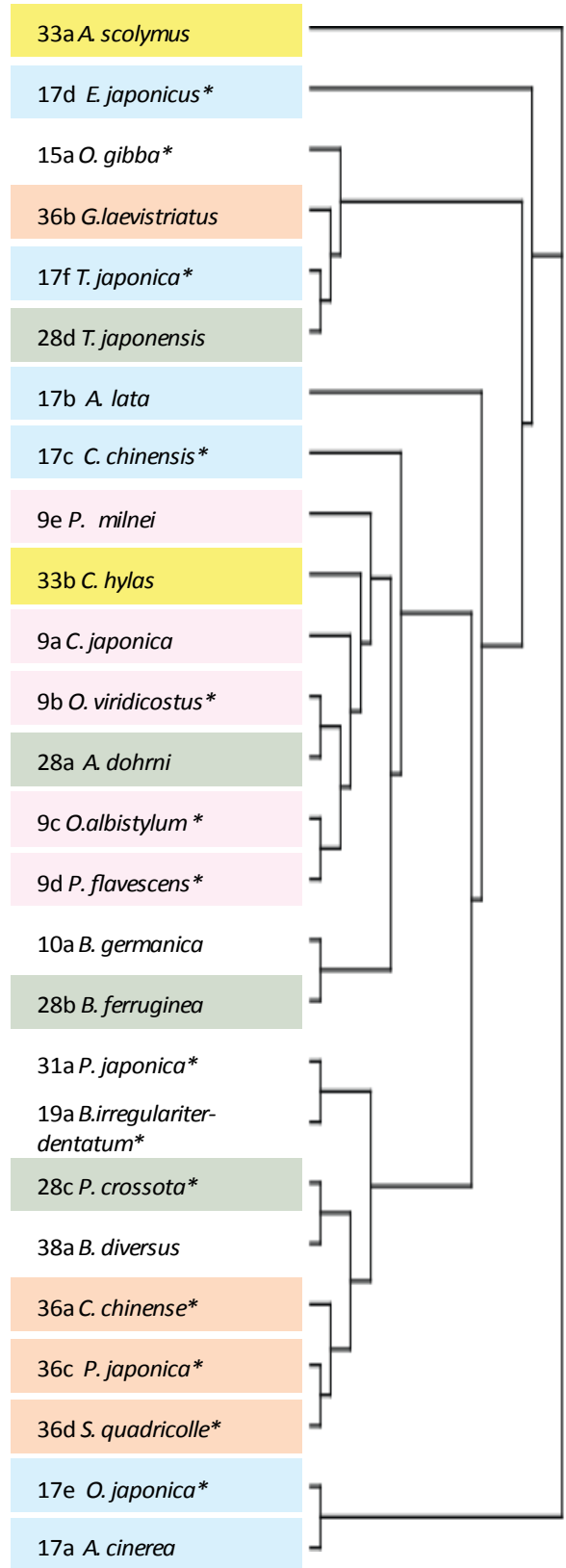

18S rDNA-based (Fig. 2b)/Object tree 
Case (- $\pi$ ) of $18 \mathrm{~S}$ rDNA-based as subject one;

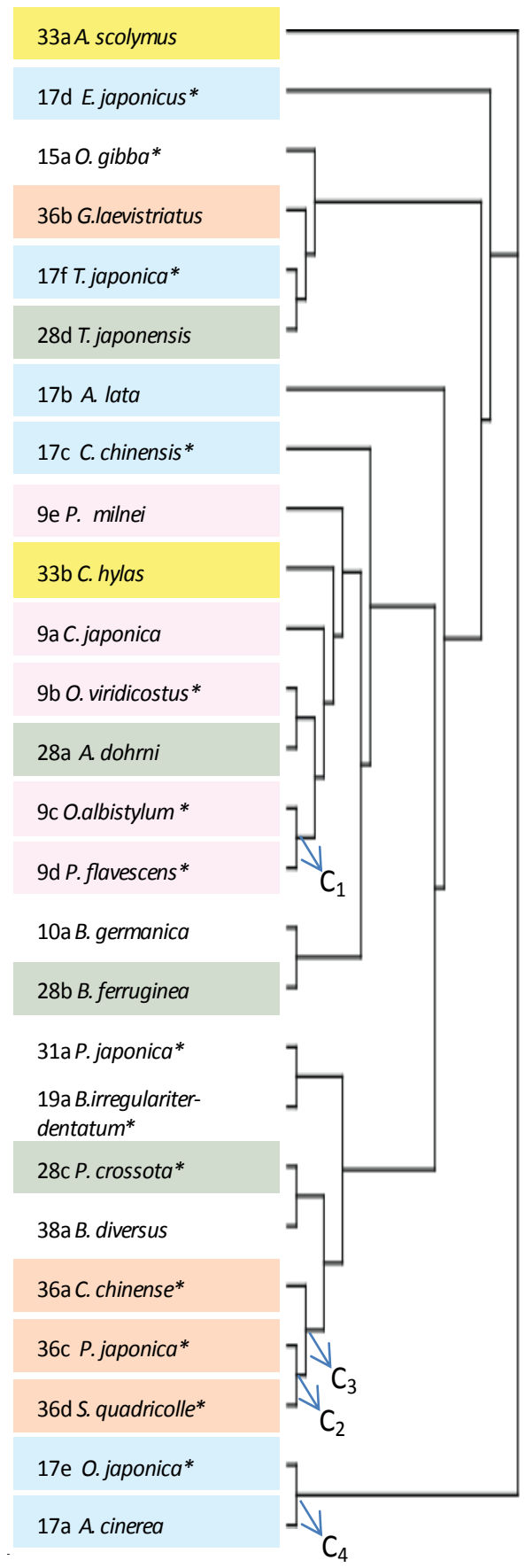

18S rDNA-based (Fig. 2b)/Subject tree

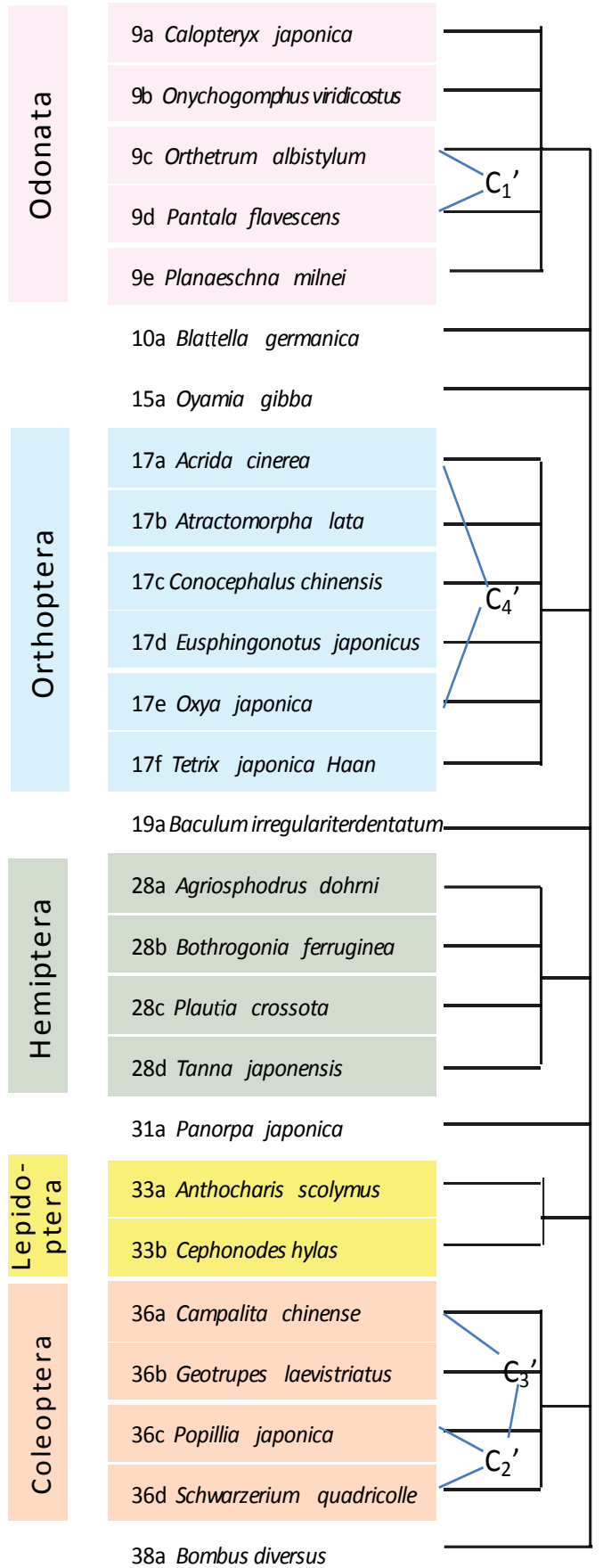

Phenotype-based/Object tree

\section{Congruence value $\left(V_{c}\right)$ :}

$$
V_{c}=0+7.5 /(2 \times 58)=0.06
$$




\subsection{Congruence value calculation between phenotype and GP-based (Fig. 3) trees.}

\section{Case $\pi$ of Phenotype-based tree as subject one;}

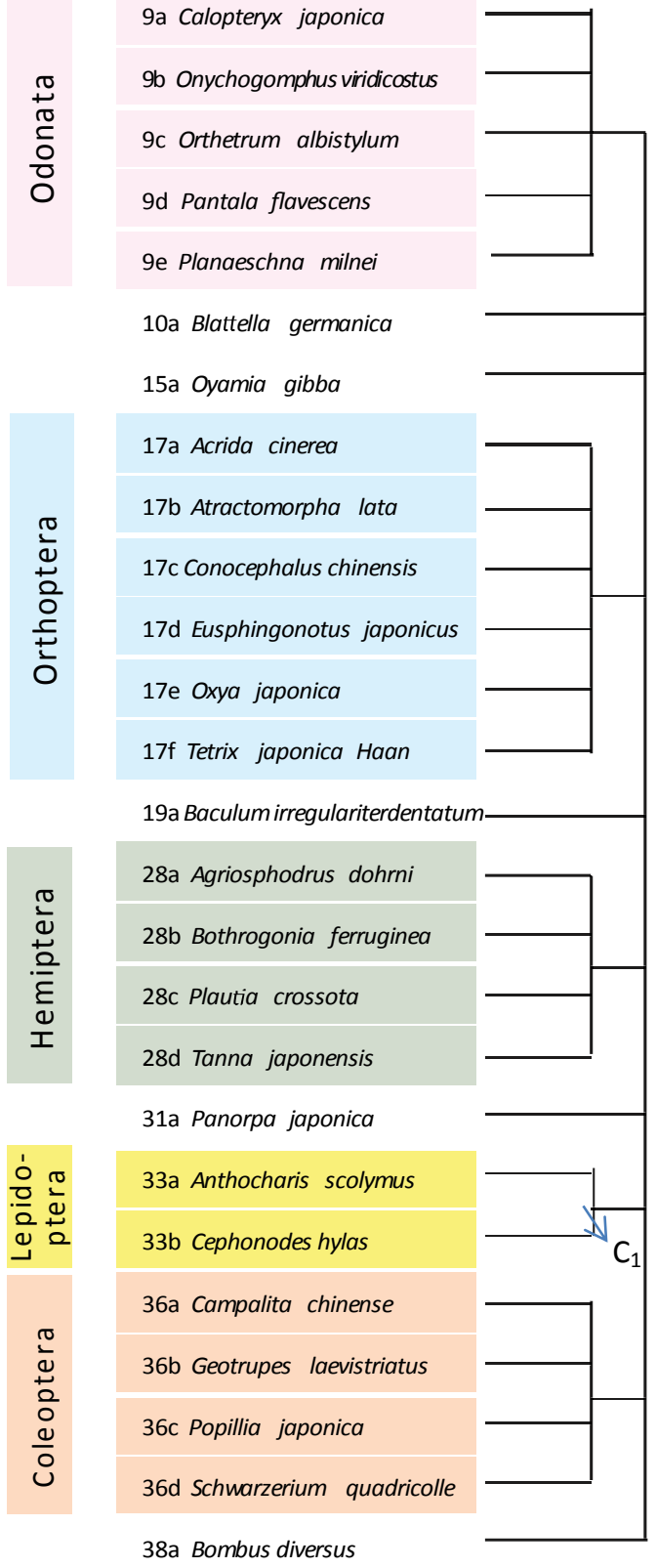

Phenotype-based/Subject tree $\begin{array}{lll}0.0 & 0.1 & 0.2\end{array}$

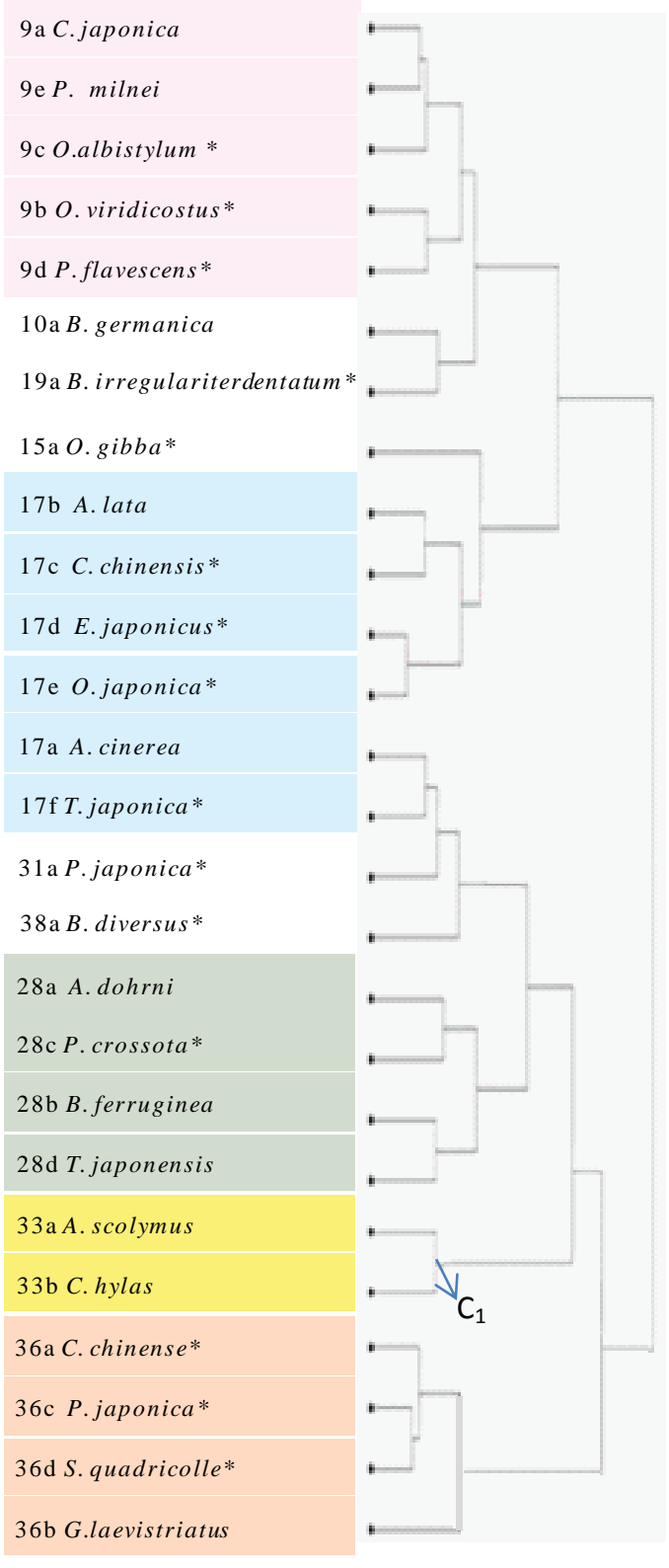

GP-based/Object tree 


\section{Case $(-\pi)$ of GP-based tree as subject one;}

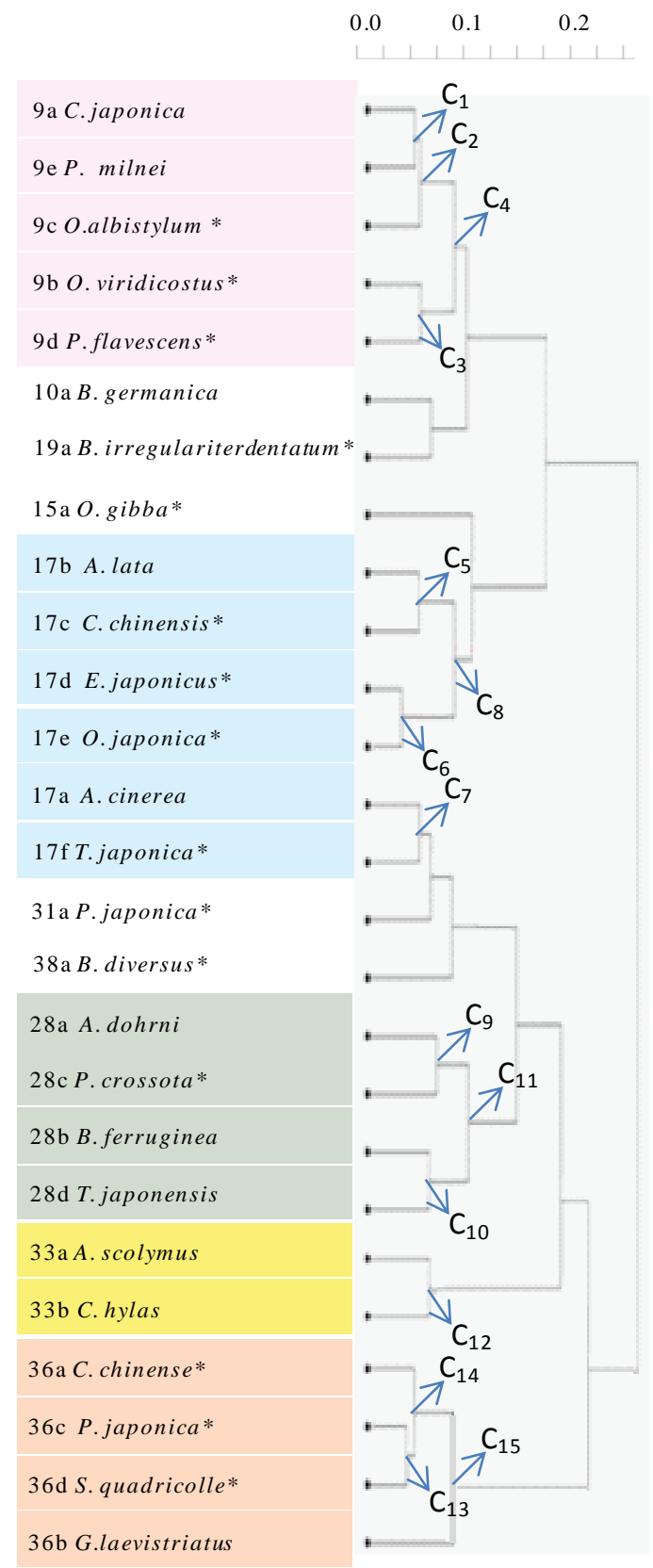

GP-based/Subject tree

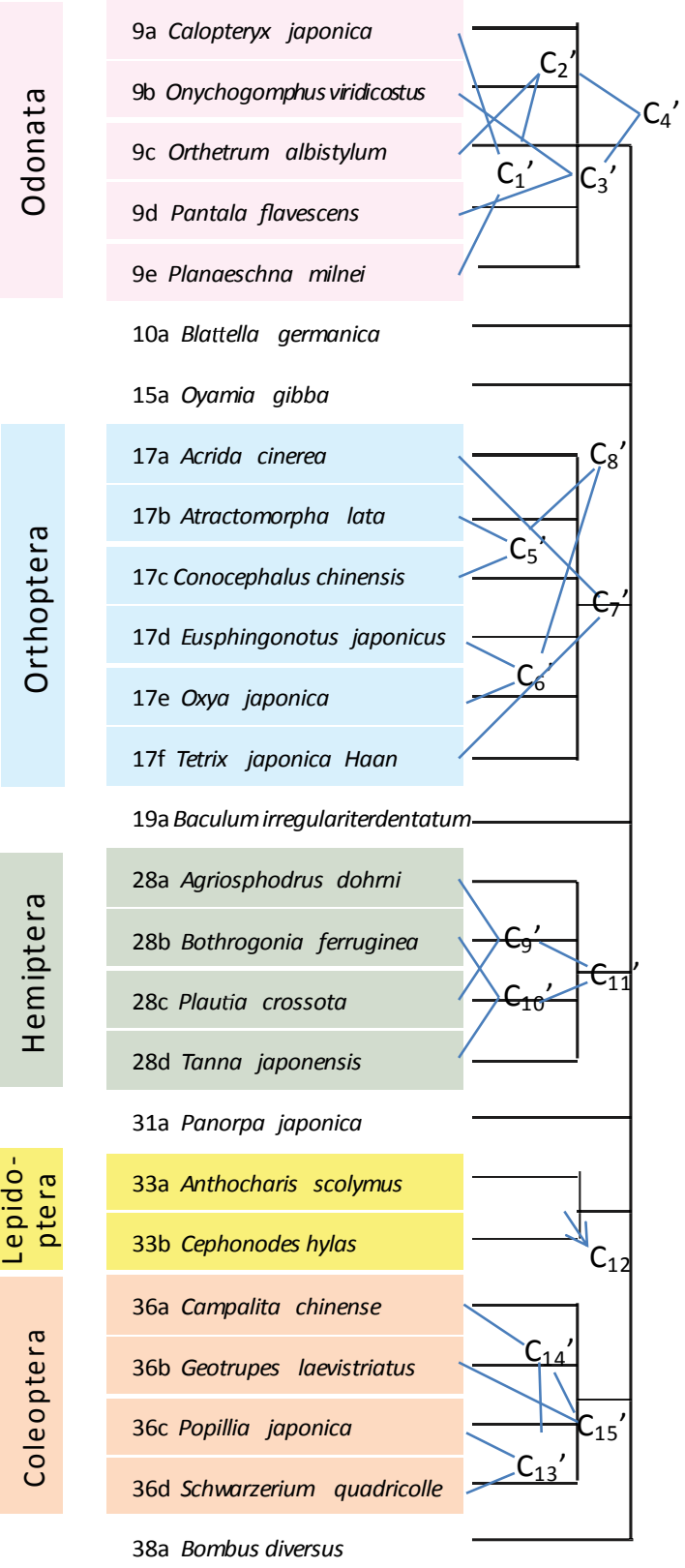

Phenotype-based/Object tree 
CMS and the number of branches for the pair of phenotype and GP-based (Fig. 3) trees:

For Case $\pi$;

\begin{tabular}{|c|c|c|c|c|}
\hline Tree name & Assigned cluster & Elements & CMS (Eq. adopted) & No. of branches \\
\hline $\begin{array}{l}\text { Phenotype-based } \\
\text { tree (subject) }\end{array}$ & $\mathrm{C}_{1}$ & $33 \mathrm{a}, 33 \mathrm{~b}$ & 2 (Eq. 1) & $=28.5$ \\
\hline $\begin{array}{c}\text { GP-based tree } \\
\text { (object) }\end{array}$ & $\mathrm{C}_{1}$ & $33 \mathrm{a}, 33 \mathrm{~b}$ & 2 (Eq. 1) & $=41.5$ \\
\hline Total & & $\mathbf{4}$ & $\mathbf{7 0}$ \\
\hline
\end{tabular}

For Case $(-\pi)$;

\begin{tabular}{|c|c|c|c|c|}
\hline Tree name & Assigned cluster & Elements & CMS (Eq. adopted) & No. of branches \\
\hline \multirow{15}{*}{$\begin{array}{l}\text { GP-based tree } \\
\text { (Subject) }\end{array}$} & $\mathrm{C}_{1}$ & $9 \mathrm{a}, 9 \mathrm{e}$ & 2(Eq. 1) & \multirow{15}{*}{$=41.5$} \\
\hline & $\mathrm{C}_{2}$ & $9 \mathrm{c}, \mathrm{C}_{1}$ & 1.5(Eq. 1) & \\
\hline & $\mathrm{C}_{3}$ & $9 \mathrm{~b}, 9 \mathrm{~d}$ & 2(Eq. 1) & \\
\hline & $\mathrm{C}_{4}$ & $\mathrm{C}_{2,}, \mathrm{C}_{3}$ & 2(Eq. 2) & \\
\hline & $\mathrm{C}_{5}$ & $17 b, 17 c$ & 2(Eq. 1) & \\
\hline & $\mathrm{C}_{6}$ & $17 \mathrm{~d}, 17 \mathrm{e}$ & 2(Eq. 1) & \\
\hline & $\mathrm{C}_{7}$ & $17 \mathrm{a}, 17 \mathrm{f}$ & 2(Eq. 1) & \\
\hline & $\mathrm{C}_{8}$ & $\mathrm{C}_{5}, \mathrm{C}_{6}$ & $1+.5=1.5$ (Eq. 2$)$ & \\
\hline & $\mathrm{C}_{9}$ & $28 \mathrm{a}, 28 \mathrm{c}$ & 2(Eq. 1) & \\
\hline & $\mathrm{C}_{10}$ & $28 \mathrm{~b}, 28 \mathrm{~d}$ & 2(Eq. 1) & \\
\hline & $\mathrm{C}_{11}$ & $\mathrm{C}_{9}, \mathrm{C}_{10}$ & 2(Eq. 2) & \\
\hline & $\mathrm{C}_{12}$ & $33 a, 33 b$ & 2(Eq. 1) & \\
\hline & $\mathrm{C}_{13}$ & $36 \mathrm{c}, 36 \mathrm{~d}$ & 2(Eq. 1) & \\
\hline & $\mathrm{C}_{14}$ & $36 \mathrm{a}, \mathrm{C}_{13}$ & 1.5(Eq. 2) & \\
\hline & $\mathrm{C}_{15}$ & $36 \mathrm{~b}, \mathrm{C}_{14}$ & 1.25 (Eq. 2) & \\
\hline \multirow{15}{*}{$\begin{array}{l}\text { Phenotype-based } \\
\text { tree (Object) }\end{array}$} & $\mathrm{C}_{1}{ }^{\prime}$ & $2(9 \mathrm{a}, 9 \mathrm{e})$ of 5 (9 series) & $<0.05$ (Eq. 3) & \multirow{15}{*}{$=28.5$} \\
\hline & $\mathrm{C}_{2}{ }^{\prime}$ & $9 \mathrm{c}, \mathrm{C}_{1}{ }^{\prime}$ & $<0.05$ (Eq. 4) & \\
\hline & $\mathrm{C}_{3}$, & $2(9 b, 9 d)$ of 5 (9 series) & $<0.05$ (Eq. 3) & \\
\hline & $\mathrm{C}_{4}{ }^{\prime}$ & $\mathrm{C}_{2}{ }^{\prime}, \mathrm{C}_{3}{ }^{\prime}$ & $<0.05$ (Eq. 3) & \\
\hline & $\mathrm{C}_{5}{ }^{\prime}$ & $2(17 b, 17 c)$ of 6 (17 series) & $<0.05$ (Eq. 3) & \\
\hline & $\mathrm{C}_{6}{ }^{\prime}$ & $2(17 \mathrm{~d}, 17 \mathrm{e})$ of 6 (17 series) & $<0.05$ (Eq. 3) & \\
\hline & $\mathrm{C}_{7}^{\prime}$ & $2(17 a, 17 f)$ of 6 (17 series) & $<0.05$ (Eq. 3) & \\
\hline & $\mathrm{C}_{8}{ }^{\prime}$ & $\mathrm{C}_{5}, \mathrm{C}_{6}{ }^{\prime}$ & $<0.05$ (Eq. 3) & \\
\hline & $\mathrm{C}_{9}{ }^{\prime}$ & $2(28 \mathrm{a}, 28 \mathrm{c})$ of 4 (28 series) & $<0.05$ (Eq. 3) & \\
\hline & $\mathrm{C}_{10}$, & $2(28 b, 28 d)$ of 4 (28 series) & $<0.05$ (Eq. 3) & \\
\hline & $\mathrm{C}_{11}{ }^{\prime}$ & $\mathrm{C}_{9}{ }^{\prime}, \mathrm{C}_{10}{ }^{\prime}$ & $<0.05$ (Eq. 3) & \\
\hline & $\mathrm{C}_{12}$ & $33 a, 33 b$ & 2(Eq. 1) & \\
\hline & $\mathrm{C}_{13}$, & $2(36 \mathrm{c}, 36 \mathrm{~d})$ of 4 (36 series) & $<0.05$ (Eq. 3) & \\
\hline & $\mathrm{C}_{14}{ }^{\prime}$ & $36 \mathrm{a}, \mathrm{C}_{13}$ & $<0.05$ (Eq. 3) & \\
\hline & $\mathrm{C}_{15}{ }^{\prime}$ & $36 \mathrm{~b}, \mathrm{C}_{14}$ ’ & $<0.05$ (Eq. 3) & \\
\hline \multicolumn{3}{|l|}{ Total } & 29.75 & 70 \\
\hline
\end{tabular}

\section{Congruence value $\left(V_{c}\right)$ :}

$$
V_{c}=(4+29.75) /(2 \times 70)=33.75 / 140=0.24
$$




\subsection{Congruence value $\left(V_{c},\right)$ between phenotype and $18 S$ rDNA-based (Fig. 2c) trees after the coarse-graining process:}

\section{Case $\pi$ of Phenotype-based tree as subject one;}

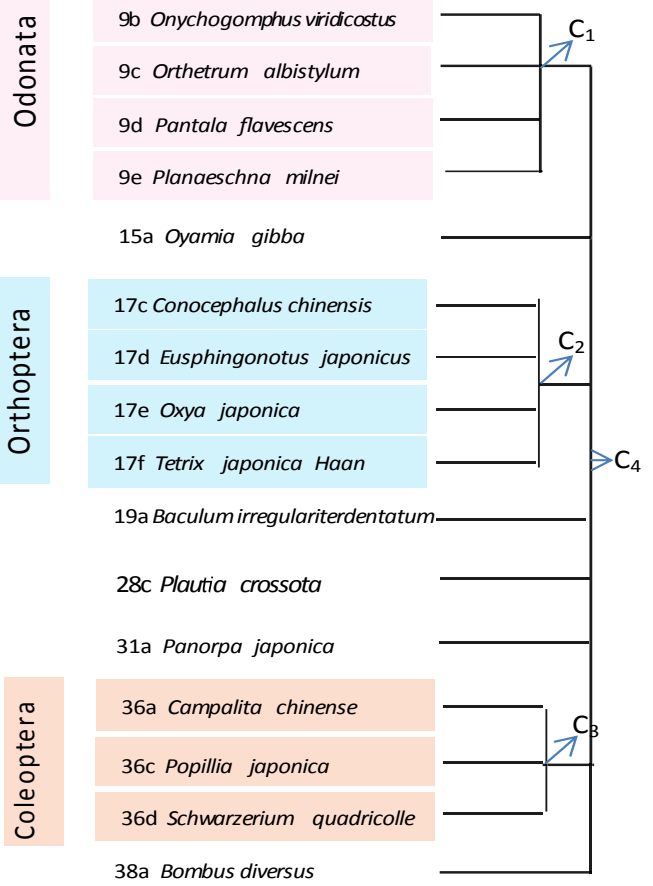

Phenotype-based/Subject tree

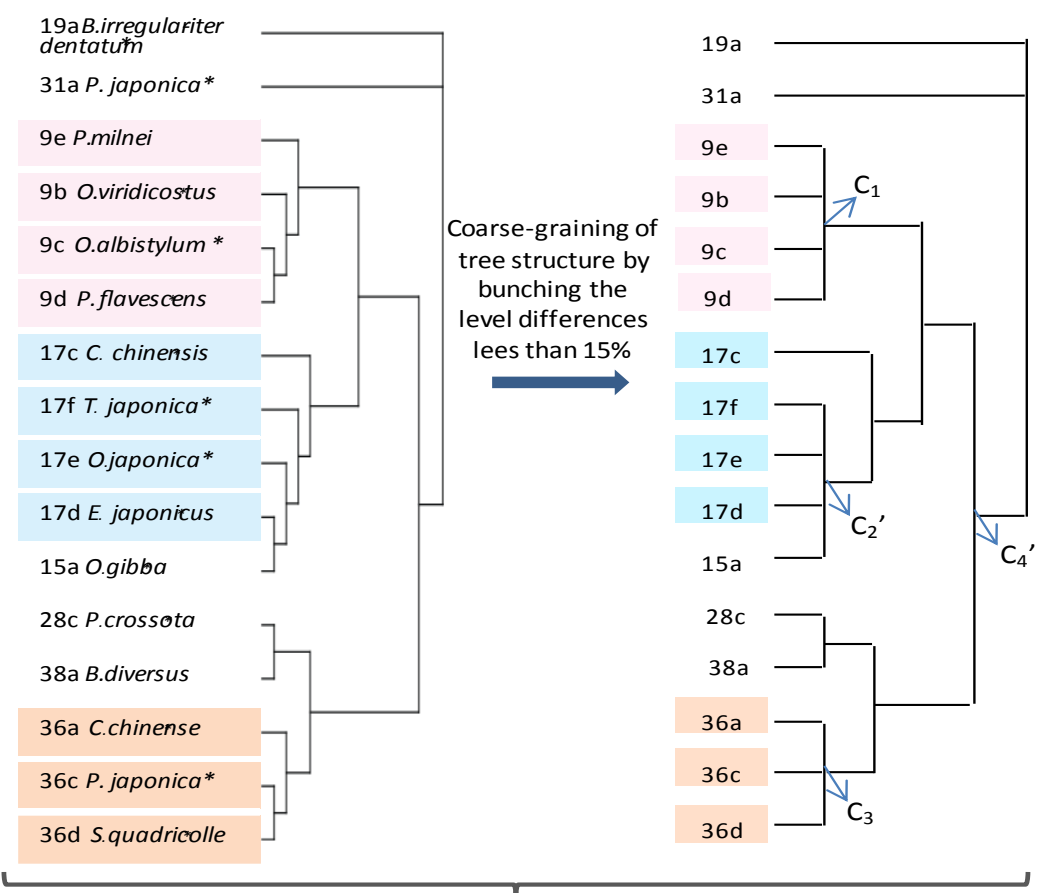

$18 S$ rDNA-based (Fig. 2c)/Object tree

\section{Case (- $\pi$ ) of 18S rDNA-based as subject one;}

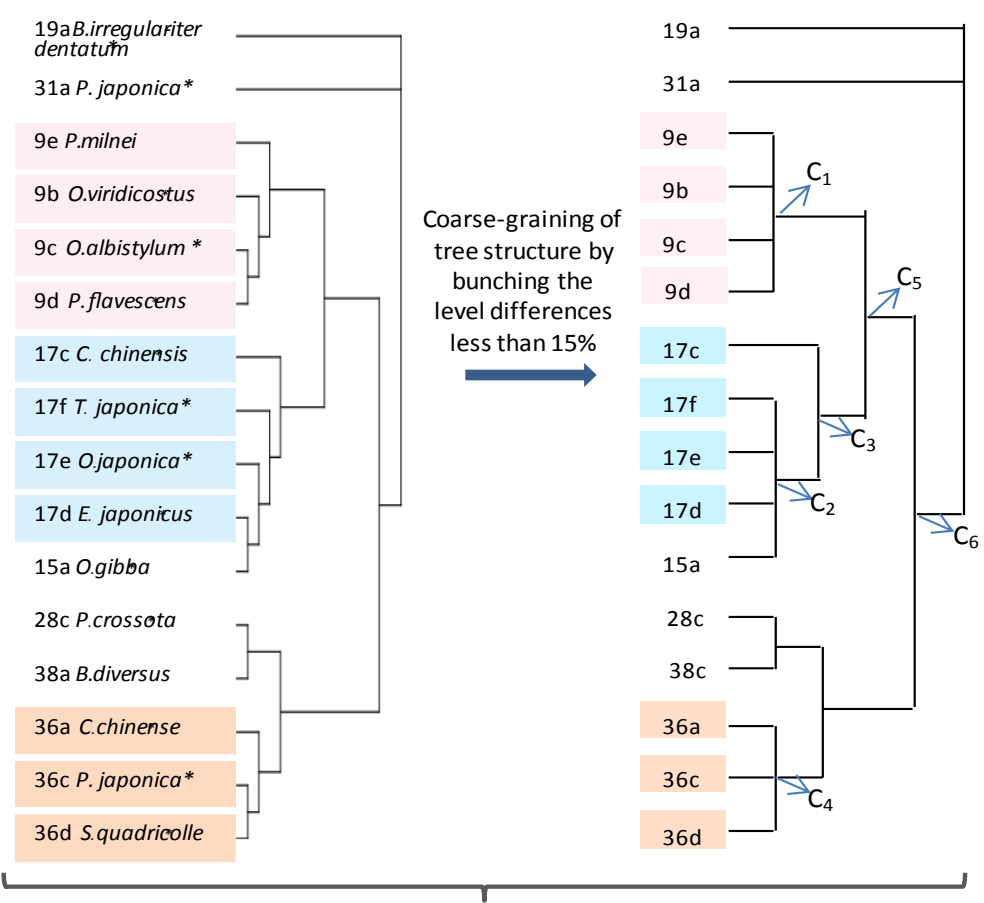

$18 S$ rDNA-based (Fig. 2c)/Subject tree

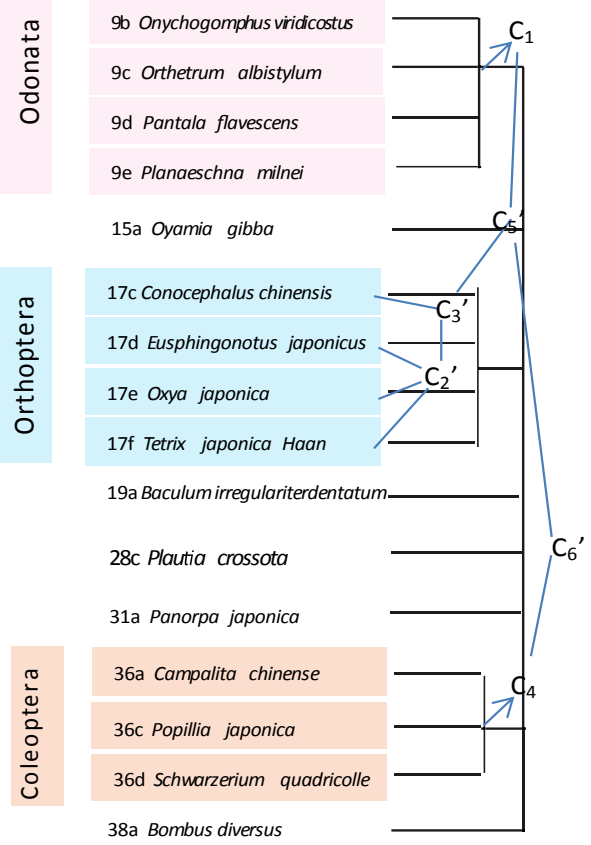

Phenotype-based/Object tree 
CMS and the number of branches for the pair of phenotype and 18S rDNA-based (Fig. 2c)

trees:

For Case $\pi$;

\begin{tabular}{|c|c|c|c|c|}
\hline Tree name & Assigned cluster & Elements & CMS (Eq. adopted) & No. of branches \\
\hline \multirow{4}{*}{$\begin{array}{l}\text { Phenotype-based } \\
\text { tree (Subject) }\end{array}$} & $\mathrm{C}_{1}$ & $9 \mathrm{~b}, 9 \mathrm{c}, 9 \mathrm{~d}, 9 \mathrm{e}$ & 4 (Eq. 1) & \multirow{4}{*}{$=16.5$} \\
\hline & $\mathrm{C}_{2}$ & $17 \mathrm{c}, 17 \mathrm{~d}, 17 \mathrm{e}, 17 \mathrm{f}$ & 4 (Eq. 1) & \\
\hline & $\mathrm{C}_{3}$ & $36 a, 36 c, 36 d$ & 3 (Eq. 1) & \\
\hline & $\mathrm{C}_{4}$ & $\begin{array}{c}\mathrm{C}_{1}, \mathrm{C}_{2}, \mathrm{C}_{3}, 15 \mathrm{a}, 19 \mathrm{a}, 28 \mathrm{c}, \\
31 \mathrm{a}, 28 \mathrm{a}\end{array}$ & 5.5 (Eq. 2) & \\
\hline \multirow{4}{*}{$\begin{array}{l}\text { 18S rDNA-based } \\
\text { tree (Object) }\end{array}$} & $\mathrm{C}_{1}$ & $9 \mathrm{e}, 9 \mathrm{~b}, 9 \mathrm{c}, 9 \mathrm{~d}$ & 4 (Eq. 1) & \multirow{4}{*}{$=20.5$} \\
\hline & $\mathrm{C}_{2}{ }^{\prime}$ & $17 \mathrm{f}, 17 \mathrm{e}, 17 \mathrm{~d}$ & $3 / 4=0.75$ (Eq. 3) & \\
\hline & $\mathrm{C}_{3}$ & $36 a, 36 c, 36 d$ & 3 (Eq. 1) & \\
\hline & $\mathrm{C}_{4}{ }^{\prime}$ & $\mathrm{C}_{1}, 17 \mathrm{e}, \mathrm{C}_{2}, 28 \mathrm{c}, 38 \mathrm{a}, \mathrm{C}_{3}$ & $\begin{array}{c}.25+.12+.25+.12+.12+.25 \\
=1.11\end{array}$ & \\
\hline \multicolumn{3}{|l|}{ Total } & 25.36 & 37 \\
\hline
\end{tabular}

For Case $(-\pi)$;

\begin{tabular}{|c|c|c|c|c|}
\hline Tree name & Assigned cluster & Elements & CMS (Eq. adopted) & No. of branches \\
\hline \multirow{6}{*}{$\begin{array}{l}\text { 18S rDNA-based } \\
\text { tree (Subject) }\end{array}$} & $\mathrm{C}_{1}$ & $9 \mathrm{e}, 9 \mathrm{~b}, 9 \mathrm{c}, 9 \mathrm{~d}$ & 4 (Eq. 1) & \multirow{6}{*}{$=20.5$} \\
\hline & $\mathrm{C}_{2}$ & $17 \mathrm{f}, 17 \mathrm{e}, 17 \mathrm{~d}, 15 \mathrm{a}$ & 4 (Eq. 1) & \\
\hline & $\mathrm{C}_{3}$ & $\mathrm{C}_{2}, 17 \mathrm{c}$ & $1+.5=1.5$ (Eq. 2$)$ & \\
\hline & $\mathrm{C}_{4}$ & $36 a, 36 c, 36 d$ & 4 (Eq. 1) & \\
\hline & $\mathrm{C}_{5}$ & $\mathrm{C}_{3}, \mathrm{C}_{1}$ & $1+.5=1.5$ (Eq. 2$)$ & \\
\hline & $\mathrm{C}_{6}$ & $\mathrm{C}_{5}, \mathrm{C}_{4}, 28 \mathrm{c}, 38 \mathrm{a}$ & $1+.25+.12+.12=1.49$ & \\
\hline \multirow{6}{*}{$\begin{array}{l}\text { Phenotype-based } \\
\text { tree (Object) }\end{array}$} & $\mathrm{C}_{1}$ & $9 \mathrm{~b}, 9 \mathrm{c}, 9 \mathrm{~d}, 9 \mathrm{e}$ & 4 (Eq. 1) & \multirow{6}{*}{$=16.5$} \\
\hline & $\mathrm{C}_{2}$, & $17 \mathrm{f}, 17 \mathrm{e}, 17 \mathrm{~d}$ & $3 / 4=0.75$ (Eq. 3) & \\
\hline & $\mathrm{C}_{3}$, & $\mathrm{C}_{2}, 17 \mathrm{c}$ & $<0.05$ & \\
\hline & $\mathrm{C}_{4}$ & $36 a, 36 c, 36 d$ & 3 (Eq. 1) & \\
\hline & $\mathrm{C}_{5}$, & $\mathrm{C}_{1}, \mathrm{C}_{3}{ }^{\prime}$ & $1+1=2($ Eq. 4$)$ & \\
\hline & $\mathrm{C}_{6}{ }^{\prime}$ & $\mathrm{C}_{5}, \mathrm{C}_{4}, 19 \mathrm{a}, 28 \mathrm{c}, 31 \mathrm{a}$ & $1+1+.5+.5+.5=3.5$ & \\
\hline \multicolumn{3}{|r|}{$-5, C_{4}, 1, a, 20 u, j 1 a$} & 28.74 & 37 \\
\hline
\end{tabular}

Congruence value $\left(V_{\mathrm{c}}{ }^{\prime}\right)$ :

$$
V_{c^{\prime}}=(25.36+28.74) /(2 \times 37)=54 / 74=0.73
$$




\subsection{Congruence value $\left(V_{c^{\prime}}\right.$ ) between phenotype and 18S rDNA-based (Fig. $2 \mathrm{~b}$ ) trees after the coarse-graining process:}

Case $\pi$ of Phenotype-based tree as subject one;

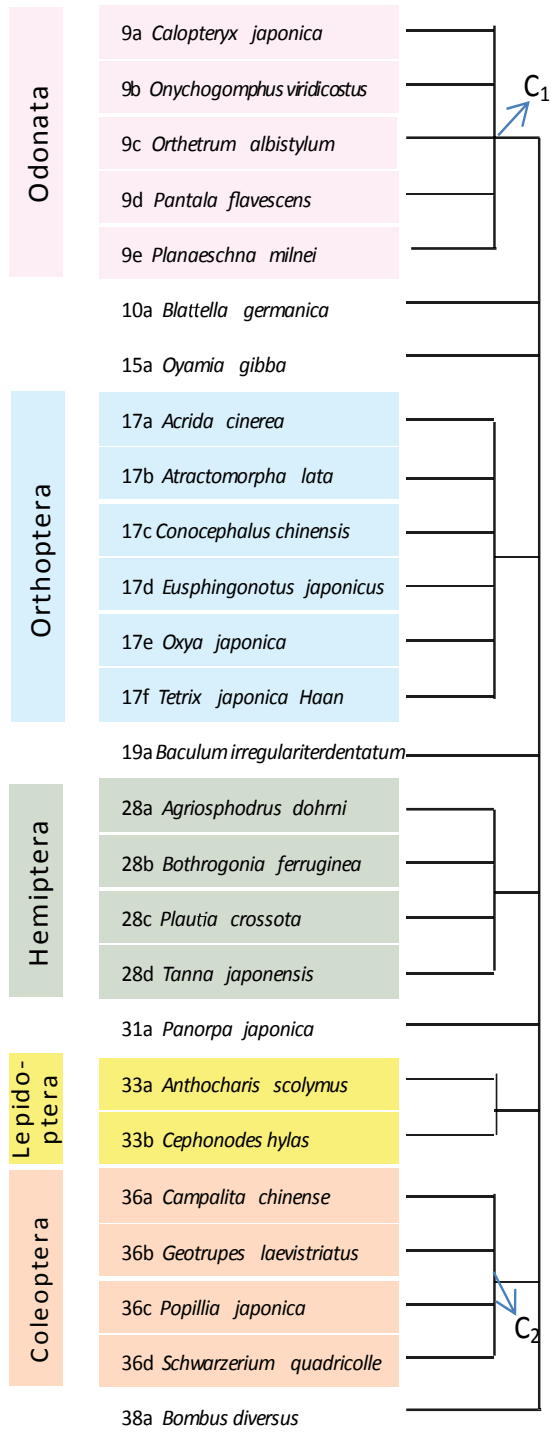

Phenotype-based/Subject tree

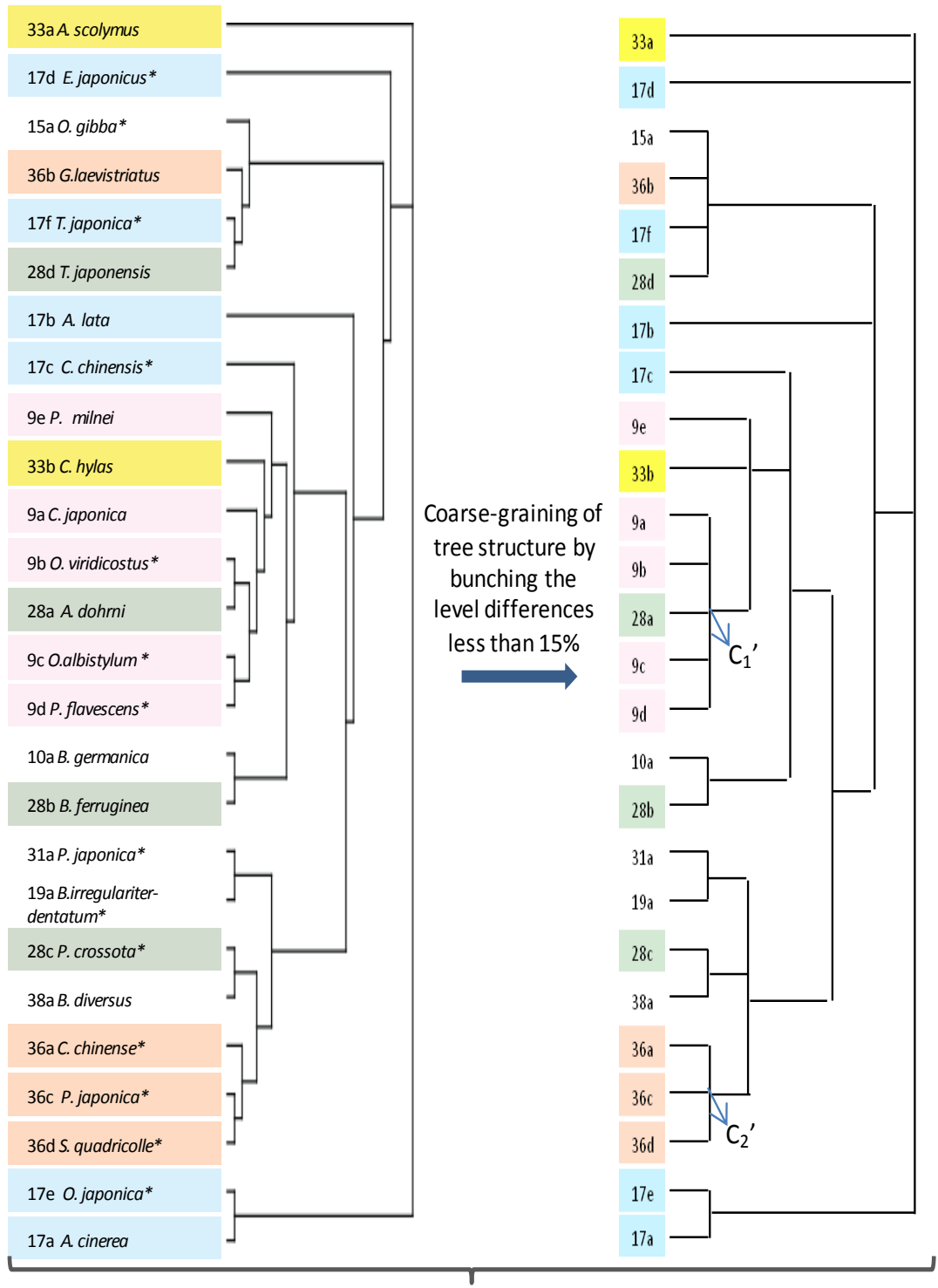

$18 \mathrm{~S}$ rDNA-based (Fig. 2b)/Object tree 


\section{Case (- $\pi$ ) of $18 \mathrm{~S}$ rDNA-based as subject one;}

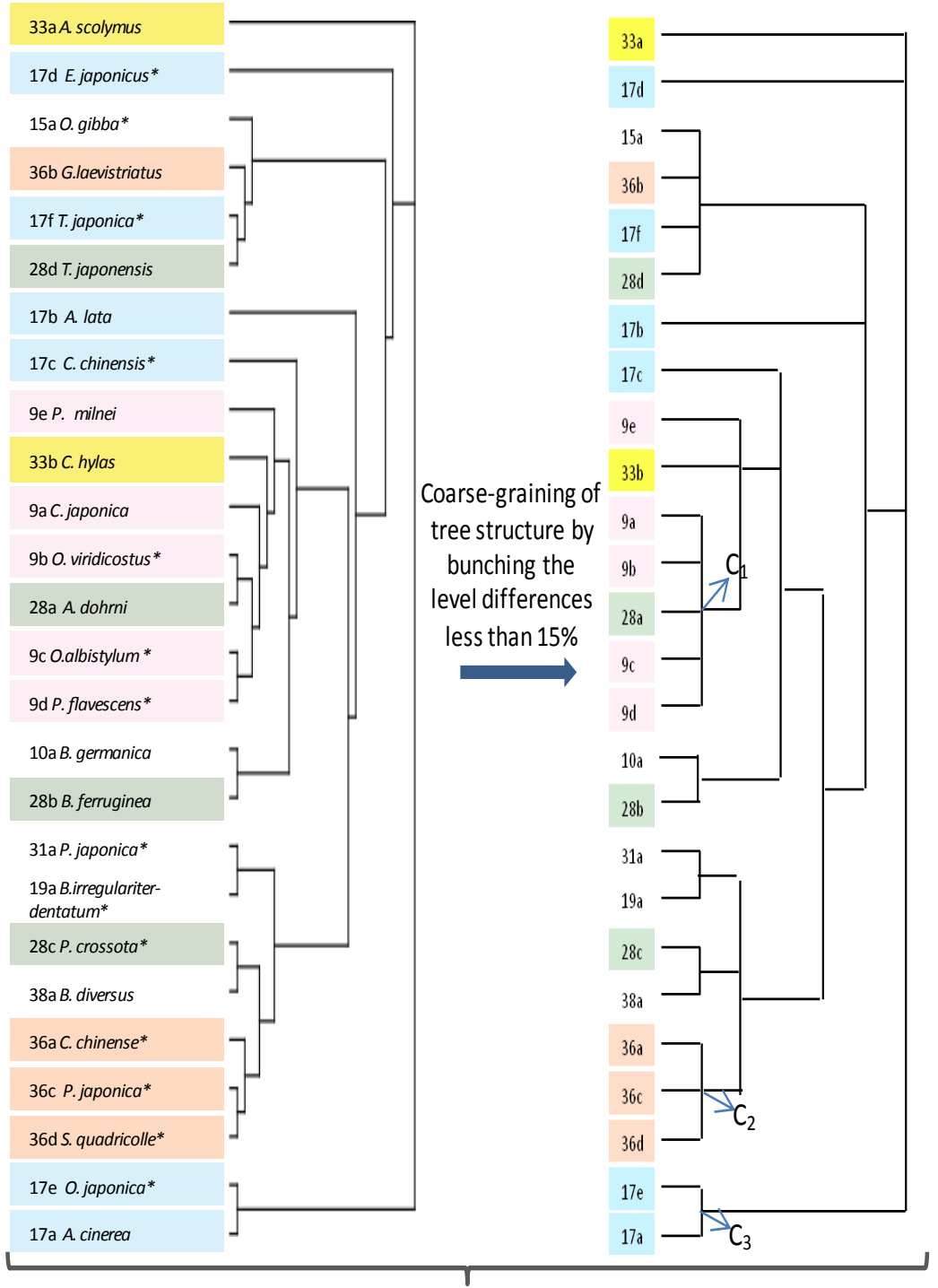

$18 \mathrm{~S}$ rDNA-based (Fig. 2b)/Subject tree

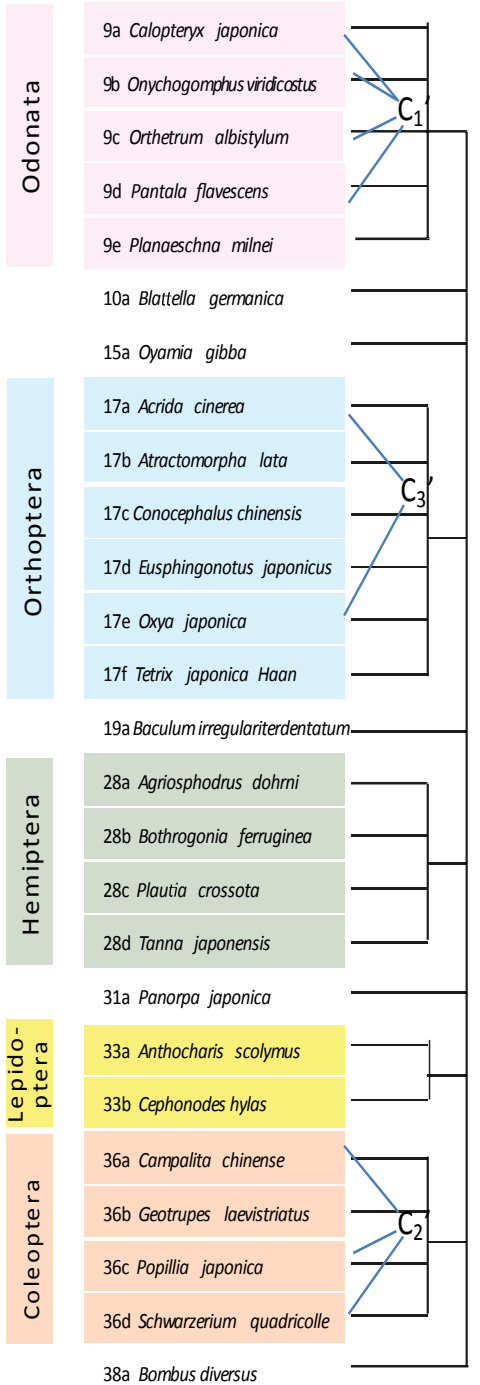

Phenotype-based/Object tree 
CMS and the number of branches for the pair of phenotype and 18S rDNA-based (Fig. 2c) trees:

\section{For Case $\pi$;}

\begin{tabular}{|c|c|c|c|c|}
\hline Tree name & Assigned cluster & Elements & CMS (Eq. adopted) & No. of branches \\
\hline \multirow{2}{*}{$\begin{array}{c}\text { Phenotype-based } \\
\text { tree (Subject) }\end{array}$} & $\mathrm{C}_{1}$ & $9 \mathrm{a}, 9 \mathrm{~b}, 9 \mathrm{c}, 9 \mathrm{~d}, 9 \mathrm{e}$ & 5 (Eq. 1) & \multirow{2}{*}{$=28.5$} \\
\hline & $\mathrm{C}_{2}$ & $36 \mathrm{a}, 36 \mathrm{~b}, 36 \mathrm{c}, 36 \mathrm{~d}$ & 4 (Eq. 1) & \\
\hline \multirow{2}{*}{$\begin{array}{l}\text { 18S rDNA-based } \\
\text { tree (Object) }\end{array}$} & $\mathrm{C}_{1}{ }^{\prime}$ & $9 a, 9 b, 9 c, 9 d$ & $5 / 4=0.8(\mathrm{Eq} .3)$ & \multirow{2}{*}{$=29.87$} \\
\hline & $\mathrm{C}_{2}{ }^{\prime}$ & $36 a, 36 c, 36 d$ & $3 / 4=0.75$ (Eq. 3) & \\
\hline \multicolumn{3}{|l|}{ Total } & $\mathbf{1 0 . 5 5}$ & 58.37 \\
\hline
\end{tabular}

For Case $(-\pi)$;

\begin{tabular}{|c|c|c|c|c|}
\hline Tree name & Assigned cluster & Elements & CMS (Eq. adopted) & No. of branches \\
\hline \multirow{3}{*}{$\begin{array}{l}\text { 18S rDNA-based } \\
\text { tree (Subject) }\end{array}$} & $\mathrm{C}_{1}$ & $9 a, 9 b, 28 a, 9 c, 9 d$ & 5 (Eq. 1) & \multirow{3}{*}{$=29.87$} \\
\hline & $\mathrm{C}_{2}$ & $36 a, 36 c, 36 d$ & 3 (Eq. 1) & \\
\hline & $\mathrm{C}_{3}$ & $17 \mathrm{e}, 17 \mathrm{a}$ & 2 (Eq. 1) & \\
\hline \multirow{3}{*}{$\begin{array}{l}\text { Phenotype-based } \\
\text { tree (Object) }\end{array}$} & $\mathrm{C}_{1}{ }^{\prime}$ & $9 a, 9 b, 9 c, 9 d$ & $4 / 5=0.8$ (Eq. 3) & \multirow{3}{*}{$=28.5$} \\
\hline & $\mathrm{C}_{2}{ }^{\prime}$ & $36 a, 36 c, 36 d$ & $3 / 4=0.75$ (Eq. 3) & \\
\hline & $\mathrm{C}_{3}^{\prime}$ & $17 \mathrm{e}, 17 \mathrm{a}$ & $<0.05$ & \\
\hline \multicolumn{3}{|l|}{ Total } & 11.55 & 58.37 \\
\hline
\end{tabular}

\section{Congruence value $\left(V_{\mathbf{c}}{ }^{\prime}\right)$ :}

$$
V_{\mathrm{c}^{\prime}}{ }^{\prime}=(10.55+11.55) /(2 \times 58.37)=22.1 / 116.7=0.19
$$




\subsection{Congruence value $\left(V_{c}^{\prime}\right)$ between phenotype and GP-based (Fig. 3) trees after the coarse-graining process:}

\section{Case $\pi$ of Phenotype-based tree as subject one;}

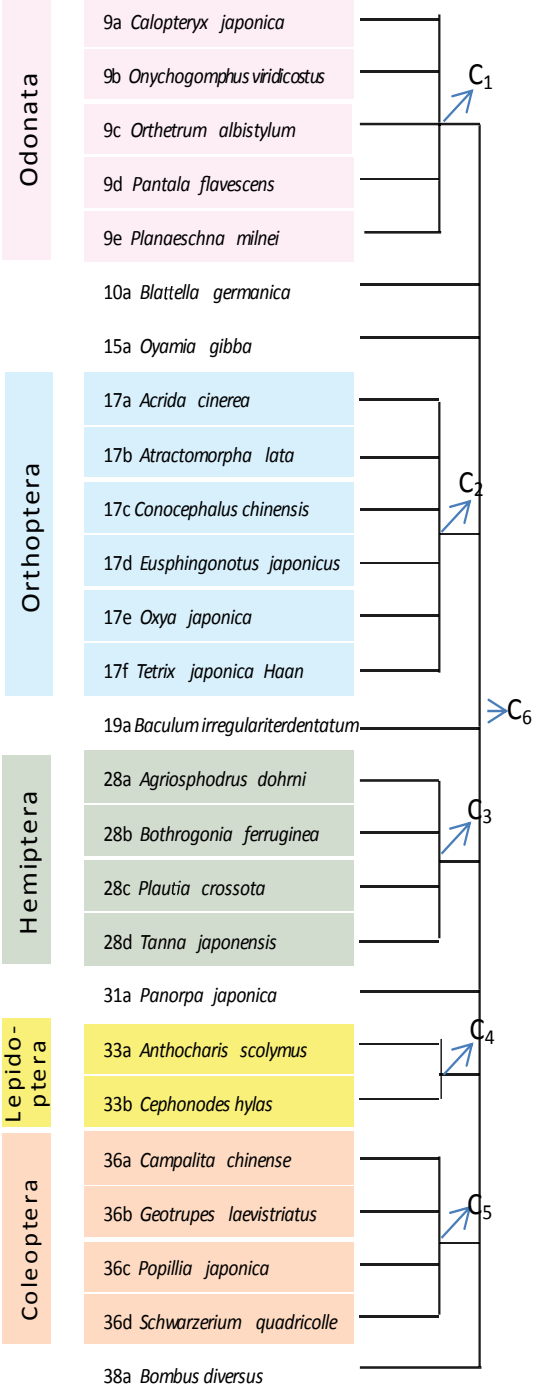

Phenotype-based/Subject tree $\begin{array}{lll}0.0 & 0.1 & 0.2\end{array}$

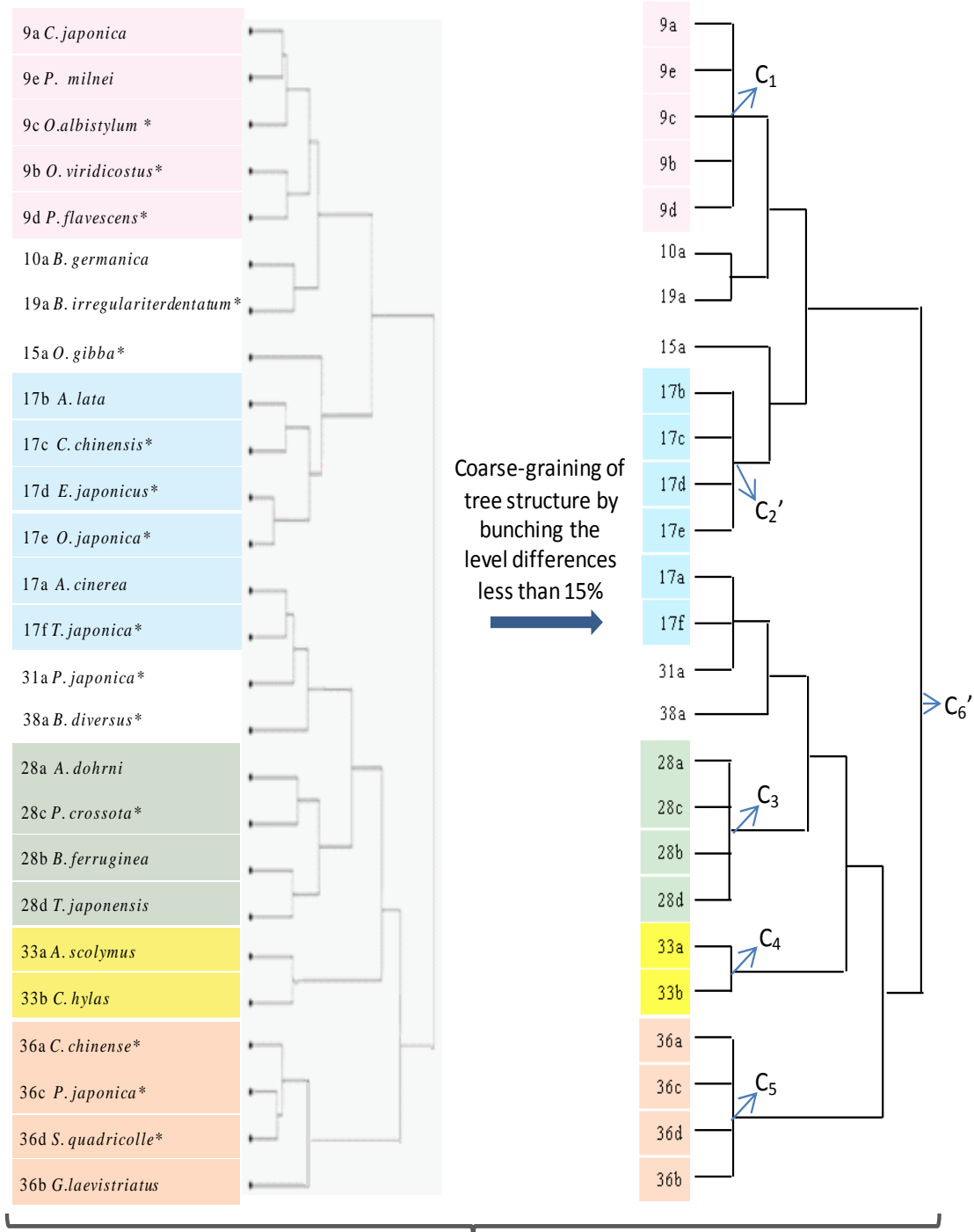

GP-based/Object tree 
Case (- $\pi$ ) of GP-based as subject one;
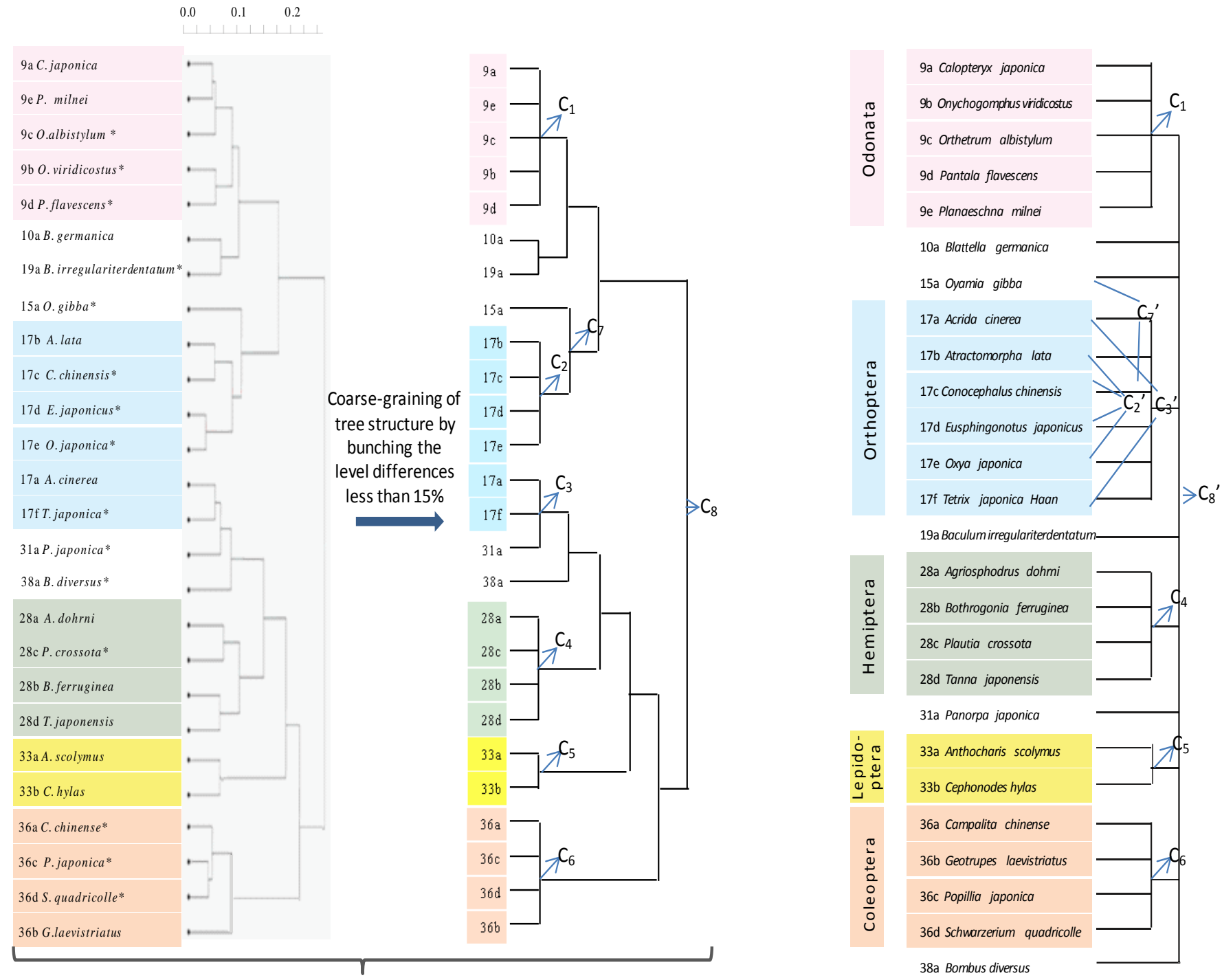

GP-based/Subject tree

Phenotype-based/Object tree 
CMS and the number of branches for the pair of phenotype and GP-based (Fig. 3) trees:

For Case $\pi$;

\begin{tabular}{|c|c|c|c|c|}
\hline Tree name & Assigned cluster & Elements & CMS (Eq. adopted) & No. of branches \\
\hline \multirow{6}{*}{$\begin{array}{l}\text { Phenotype-based } \\
\text { tree (Subject) }\end{array}$} & $\mathrm{C}_{1}$ & $9 \mathrm{a}, 9 \mathrm{~b}, 9 \mathrm{c}, 9 \mathrm{~d}, 9 \mathrm{e}$ & 5 (Eq. 1) & \multirow{6}{*}{$=28.5$} \\
\hline & $\mathrm{C}_{2}$ & $17 \mathrm{a}, 17 \mathrm{~b}, 17 \mathrm{c}, 17 \mathrm{~d}, 17 \mathrm{e}, 17 \mathrm{f}$ & 6 (Eq. 1) & \\
\hline & $\mathrm{C}_{3}$ & $28 \mathrm{a}, 28 \mathrm{~b}, 28 \mathrm{c}, 28 \mathrm{~d}$ & 4 (Eq. 1) & \\
\hline & $\mathrm{C}_{4}$ & $33 a, 33 b$ & 2 (Eq. 1) & \\
\hline & $\mathrm{C}_{5}$ & $36 a, 36 b, 36 c, 36 d$ & 4 (Eq. 1) & \\
\hline & $\mathrm{C}_{6}$ & 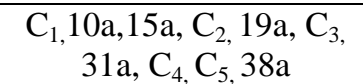 & $\begin{array}{c}1+.5+.5+1+.5+1+.5+1+1+ \\
.5=7.5\end{array}$ & \\
\hline \multirow{6}{*}{$\begin{array}{l}\text { GP-based tree } \\
\quad \text { (Object) }\end{array}$} & $\mathrm{C}_{1}$ & $9 \mathrm{a}, 9 \mathrm{~b}, 9 \mathrm{c}, 9 \mathrm{~d}, 9 \mathrm{e}$ & 5 (Eq. 1) & \multirow{6}{*}{$=36.12$} \\
\hline & $\mathrm{C}_{2}{ }^{\prime}$ & $17 \mathrm{~b}, 17 \mathrm{c}, 17 \mathrm{~d}, 17 \mathrm{e}$ & $4 / 30=0.13$ (Eq. 3) & \\
\hline & $\mathrm{C}_{3}$ & $28 \mathrm{a}, 28 \mathrm{~b}, 28 \mathrm{c}, 28 \mathrm{~d}$ & 4 (Eq. 1) & \\
\hline & $\mathrm{C}_{4}$ & $33 a, 33 b$ & 2 (Eq. 1) & \\
\hline & $\mathrm{C}_{5}$ & 36a, 36b, 36c, 36d & 4 (Eq. 1) & \\
\hline & $\mathrm{C}_{6}{ }^{\prime}$ & $\begin{array}{c}\mathrm{C}_{1}, 10 \mathrm{a}, 19 \mathrm{a}, 15 \mathrm{a}, \mathrm{C}_{2}, 17 \mathrm{a}, \\
17 \mathrm{f}, 31 \mathrm{a}, 38 \mathrm{a}, \mathrm{C}_{3}, \mathrm{C}_{4}, \mathrm{C}_{5}\end{array}$ & $<.05+<.05 \ldots \ldots \ldots$ & \\
\hline \multicolumn{3}{|l|}{ Total } & 43.63 & 64.62 \\
\hline
\end{tabular}

For Case $(-\pi)$;

\begin{tabular}{|c|c|c|c|c|}
\hline Tree name & Assigned cluster & Elements & CMS (Eq. adopted) & No. of branches \\
\hline \multirow{8}{*}{$\begin{array}{l}\text { GP-based tree } \\
\quad \text { (Subject) }\end{array}$} & $\mathrm{C}_{1}$ & $9 \mathrm{a}, 9 \mathrm{~b}, 9 \mathrm{c}, 9 \mathrm{~d}, 9 \mathrm{e}$ & 5 (Eq. 1) & \multirow{8}{*}{$=36.12$} \\
\hline & $\mathrm{C}_{2}$ & $17 \mathrm{~b}, 17 \mathrm{c}, 17 \mathrm{~d}, 17 \mathrm{e}$ & 4 (Eq. 1) & \\
\hline & $\mathrm{C}_{3}$ & $17 \mathrm{a}, 17 \mathrm{f}, 31 \mathrm{a}$ & 3 (Eq. 1) & \\
\hline & $\mathrm{C}_{4}$ & $28 \mathrm{a}, 28 \mathrm{c}, 28 \mathrm{~b}, 28 \mathrm{~d}$ & 4 (Eq. 1) & \\
\hline & $\mathrm{C}_{5}$ & $33 a, 33 b$ & 2 (Eq. 1) & \\
\hline & $\mathrm{C}_{6}$ & $36 a, 36 c, 36 d, 36 b$ & 4 (Eq. 1) & \\
\hline & $\mathrm{C}_{7}$ & $\mathrm{C}_{2}, 15 \mathrm{a}$ & $1+.5=1.5$ (Eq. 2$)$ & \\
\hline & $\mathrm{C}_{8}$ & $\begin{array}{c}\mathrm{C}_{1,}, 10 \mathrm{a}, 19 \mathrm{a}, \mathrm{C}_{7}, \mathrm{C}_{2}, \mathrm{C}_{3}, \\
38 \mathrm{a}, \mathrm{C}_{4}, \mathrm{C}_{5}, \mathrm{C}_{6}\end{array}$ & $\sim 1.0$ (Eq. 2 ) & \\
\hline \multirow{8}{*}{$\begin{array}{l}\text { Phenotype-based } \\
\text { tree (Object) }\end{array}$} & $\mathrm{C}_{1}$ & $9 \mathrm{a}, 9 \mathrm{~b}, 9 \mathrm{c}, 9 \mathrm{~d}, 9 \mathrm{e}$ & 5 (Eq. 1) & \multirow{8}{*}{$=28.5$} \\
\hline & $\mathrm{C}_{2}{ }^{\prime}$ & $17 \mathrm{~b}, 17 \mathrm{c}, 17 \mathrm{~d}, 17 \mathrm{e}$ & $4 / 30=0.13$ (Eq. 3) & \\
\hline & $\mathrm{C}_{3}{ }^{\prime}$ & $17 \mathrm{a}, 17 \mathrm{f}$ & $<0.05$ & \\
\hline & $\mathrm{C}_{4}$ & $28 \mathrm{a}, 28 \mathrm{~b}, 28 \mathrm{c}, 28 \mathrm{~d}$ & 4 (Eq. 1) & \\
\hline & $\mathrm{C}_{5}$ & $33 a, 33 b$ & 2 (Eq. 1) & \\
\hline & $\mathrm{C}_{6}$ & $36 a, 36 b, 36 c, 36 d$ & 4 (Eq. 1) & \\
\hline & $\mathrm{C}_{7}{ }^{\prime}$ & $15 \mathrm{a}, \mathrm{C}_{2}$, & $1+.5=1.5($ Eq. 2$)$ & \\
\hline & $\mathrm{C}_{8}{ }^{\prime}$ & $\begin{array}{c}\mathrm{C}_{1}, 10 \mathrm{a}, 15 \mathrm{a}, \mathrm{C}_{2}, \mathrm{C}_{3}, \\
\mathrm{C}_{7}, \mathrm{C}_{4}, \mathrm{C}_{5}, \mathrm{C}_{6}, 38 \mathrm{a},\end{array}$ & $\begin{array}{c}1+.5+.5+1+1+1+1+1+1+. \\
5=8.5\end{array}$ & \\
\hline \multicolumn{3}{|l|}{ Total } & 47.13 & 64.62 \\
\hline
\end{tabular}

Congruence value $\left(V_{\mathrm{c}}{ }^{\prime}\right)$ :

$V_{\mathrm{c}}{ }^{\prime}=(43.63+47.13) /(2 \times 64.62)=91.8 / 129=0.71$ 
Summary findings of the current study:

\begin{tabular}{|c|c|c|}
\hline Trees & $V_{c}$ & $V_{c}{ }^{\prime}$ \\
\hline $\begin{array}{c}\text { Phenotype vs 18S rDNA-based tree } \\
\text { (Fig. 2b, 26 samples) }\end{array}$ & 0.06 & 0.19 \\
\hline $\begin{array}{c}\text { Phenotype vs 18S rDNA-based tree } \\
\text { (Fig. 2c, 16 samples) }\end{array}$ & 0.26 & 0.73 \\
\hline Phenotype vs GP-based tree (Fig. 3) & 0.24 & 0.71 \\
\hline
\end{tabular}

